\title{
Identification of a Synergistic Multi-Drug Combination Active in Cancer Cells via the Prevention of Spindle Pole Clustering
}

\author{
Andrea Weiss ${ }^{1,2,+}{ }^{\text {, Morgan Le Roux-Bourdieu }}{ }^{2,3,+}{ }^{\mathbb{D}}$, Marloes Zoetemelk ${ }^{1,2}$, George M. Ramzy ${ }^{1}$, \\ Magdalena Rausch ${ }^{1,2}$, Daniela Harry ${ }^{3}$, Marijana Miljkovic-Licina ${ }^{2,4}$, Katayoun Falamaki ${ }^{3}$, \\ Bernard Wehrle-Haller ${ }^{2,3}$, Patrick Meraldi ${ }^{2,3}$ and Patrycja Nowak-Sliwinska 1,2,*(D) \\ 1 Institute of Pharmaceutical Sciences of Western Switzerland, Faculty of Sciences, University of Geneva, \\ 1 Rue Michel-Servet, CMU, 1211 Geneva 4, Switzerland \\ 2 Translational Research Centre in Oncohaematology, 1 Rue Michel-Servet, CMU, 1211 Geneva 4, Switzerland \\ 3 Department of Cell Physiology and Metabolism, University of Geneva Medical School, 1 Rue Michel-Servet, \\ CMU, 1211 Geneva 4, Switzerland \\ 4 Department of Pathology and Immunology, University of Geneva Medical School, 1 Rue Michel-Servet, \\ CMU, 1211 Geneva 4, Switzerland \\ * Correspondence: Patrycja.Nowak-Sliwinska@unige.ch; Tel.: +41-22-379-3352 \\ + These authors contributed equally to this study.
}

Received: 7 October 2019; Accepted: 16 October 2019; Published: 22 October 2019

\begin{abstract}
A major limitation of clinically used cancer drugs is the lack of specificity resulting in toxicity. To address this, we performed a phenotypically-driven screen to identify optimal multidrug combinations acting with high efficacy and selectivity in clear cell renal cell carcinoma (ccRCC). The search was performed using the Therapeutically Guided Multidrug Optimization (TGMO) method in ccRCC cells (786-O) and nonmalignant renal cells and identified a synergistic low-dose four-drug combination (C2) with high efficacy and negligible toxicity. We discovered that $\mathbf{C} 2$ inhibits multipolar spindle pole clustering, a survival mechanism employed by cancer cells with spindle abnormalities. This phenotype was also observed in 786-O cells resistant to sunitinib, the first line ccRCC treatment, as well as in melanoma cells with distinct percentages of supernumerary centrosomes. We conclude that C2-treatment shows a high efficacy in cells prone to form multipolar spindles. Our data suggest a highly effective and selective C2 treatment strategy for malignant and drug-resistant cancers.
\end{abstract}

Keywords: drug combinations; drug synergy; centrosome; multipolar spindle pole clustering

\section{Introduction}

Combination therapies represent an attractive treatment option due to their potential to overcome drug resistance by targeting non-overlapping signaling pathways, thereby decreasing the probability of developing cross-resistance [1]. While off-target toxicities are a major concern of new drug combinations, synergistic drug combinations can achieve higher therapeutic selectivity than single drugs [2]. Causally, this can be explained by various phenomena. If properly designed, synergistic drug combinations can (i) enable targeting of deregulated networks from multiple angles [3], thereby challenging the robust and compensatory nature of complex biological systems [4,5], and (ii) allow for effective therapies with reduced toxicity by employing lower single drug doses of compounds with non-overlapping dose-related toxicities [6,7]. However, a major limitation of clinically used cancer drugs is the lack of specificity resulting in toxicity. 
The use of properly tailored drug combinations is a promising alternative to overcome the above-mentioned limitations of cancer therapy. The limited success of clinically tested drug combinations, thus far, can largely be attributed to intolerable toxicities, lack of adequate efficacy and acquired drug resistance. This may be a result of current clinical practice where drug combination components are selected empirically or based on the success of single-drug therapies. Subsequently, the drugs combined are not selected for their synergistic or selective activity. To address these issues, various drug optimization methods have been developed [8,9]. One of the major challenges in defining optimal drug cocktails is the immense number of combinatorial possibilities. Not only must the selection of candidate drugs be taken into consideration, but also their relative dose ratios, their potential for synergisms or antagonisms, and their toxicity profiles. Already combining 10 drugs at six doses represents $6^{10}$, i.e., over 60 million, possible drug-dose ratio combinations.

In order to solve these problems, we have previously developed the streamlined-Feedback System Control (s-FSC) technology, a strategy for rapid in vitro identification of optimized, synergistic low-dose multi-drug combinations [8-11]. In contrast to other approaches based on pharmacogenomics or high-throughput screening, this phenotypically-driven method identifies synergistic drug mixtures with minimal experimental effort. Moreover, it does not require background and mechanistic information about the system prior to the start of optimization. The s-FSC is based on the use of response surface methodology and an orthogonal array composite design (OACD) approach with linear regression analysis $[8,9,12]$. It selectively samples a minimal number of experimental data points in order to create the cell's response surface, i.e., drug dose-efficacy dependence in terms of second-order linear regression models that are used to select synergistic drug interactions [9].

In this study, we used for the first time the Therapeutically Guided Multidrug Optimization (TGMO) method that is based on the s-FSC technique. In the TGMO approach, drug combinations are tested simultaneously on both cancer (in this case human clear cell renal cell carcinoma cell line, 786-O) and non-cancerous cells (e.g., nonmalignant renal cells, in this case HEK-293T cells) at low doses (i.e., below maximal plasma doses, MPD, in humans). Therefore, this strategy allows the identification of optimized multidrug combinations (ODC) acting with high efficacy and selectivity. We carried out our study in ccRCC, because it is the most aggressive and common type of kidney cancer, representing about $70 \%$ of kidney tumors [13]. This selection was also dictated by the chemo-and radio-resistant properties of ccRCC and the fact that the success of pharmacological intervention with kinase inhibitors (KI) and histone deacetylase inhibitors (HDACI) has been proven in the clinic. Moreover, many HDACI sensitize cancer cells to both cytotoxic and targeted agents [14]. Various clinical and preclinical data suggest synergistic interactions between KI and HDACI [15-18].

Starting from a set of four HDACI and six KI, using the TGMO method we identified a selective optimal drug combination (ODC) after testing $0.37 \%$ of all possible experimental conditions accompanied by modeling-based data analysis. The investigation on the mechanism of action of the ODC revealed the induction of cell cycle arrest, actin cytoskeleton disruption, and dysfunctional cell divisions due to persistent aberrant mitotic spindle structures.

\section{Results}

\subsection{Therapeutically Guided Multidrug Optimization Screen Identifies Synergistic Low-Dose Drug Combinations Consisting of HDAC and Kinase Inhibitors}

A set of ten compounds, which included four HDAC inhibitors, five tyrosine kinase inhibitors (TKI) inhibitors, and one serine-threonine KI (Table 1 and Supplementary Materials) was selected for the Therapeutically Guided Multidrug Optimization (TGMO)-based screen (Figure 1).

The TGMO search was initiated by generating dose-response curves for each drug (Supplementary Figure S1). The experiments were performed in a simple in vitro cell viability bioassay (metabolic activity in ATP levels), using the human clear cell renal cell carcinoma (ccRCC) 786-O cell line and the nonmalignant human renal (HEK-293T) cells. 786-O cells were selected based on their lack of sensitivity to $\mathrm{HDACI}$ and known resistance to sunitinib, the first line treatment for $\mathrm{ccRCC}$. The $\mathrm{ED}_{50}$ dose (the 
dose that inhibited $50 \%$ of cell viability, as compared to the control) of each drug was determined by fitting dose-response data on a semi-log scale. The $\mathrm{ED}_{20}$ (or lower, based on clinically attainable drug plasma concentrations) was selected as the highest dose for combinatorial drug screening (Table 1). The second drug dose used in the screen was half of the $\mathrm{ED}_{20}$.

Drug combinations for experimental testing were defined by an orthogonal array composite design (OACD) [12] (Figure 1a,II and Supplementary Materials). This methodology defines a set of experimental points to be tested selected from all possible combinations. During the experiments (Figure 1a,III), the efficacy in the therapeutic window (TW, the difference in efficacy between HEK-293T and 786-O cells) was used to generate second-order linear regression models (Figure 1a,IV, efficacy in red bars and TW in blue bars, Supplementary Figure S2a).

For each regression model, an estimated regression coefficient was generated for the significant terms in the model, including "single-drug first order", "two-drug interaction", and "single-drug second-order". These terms describe the contribution of each drug as an individual agent and as a drug pair to the overall activity of the drug combinations. The accuracy and reliability of the regression models were assessed based on residual analysis (Supplementary Figure S2b). The generated models made it possible to analyze drug-drug interactions and to identify synergistic, selective drug interactions, guiding further drug selection and elimination. This facilitated refinement of the drugs to be tested in the subsequent experimental rounds and the selection of a drug combination with maximal cancer cell viability inhibition and minimal toxicity in noncancerous cells (Figure 1a,V).

In Search 1, 91 drug combinations were screened. Stepwise regression analysis of the experimental data led to the elimination of three drugs, i.e., LBH-589, SAHA, and axitinib, due to the lack of synergistic interaction and/or no statistical significance in single drug first-order terms (Figure 1b). In Search 2, we tested a set of 50 combinations composed of up to seven drugs (Figure 1c,d) along with a set of 25 four-drug combinations (Figure 1e). The four-drug combinations selected based on Search 1 were comprised of CI-994, tubacin, erlotinib, and dasatinib. Search 3 (Figure 1e) evaluated other promising four-drug combinations identified in the seven-drug screen (Search 2). The synergy between tubacin and erlotinib was confirmed in all three of the four-drug sets investigated. The two four-drug sets investigated in Search 3 did not show improved efficacy over the original four-drug combination screened in Search 2, as synergy was driven by the same interaction between tubacin and erlotinib and first-order effects were not superior to the primary selection. Thus, the TGMO method identified a selective ODC, from here on called C1, consisting of CI-994, tubacin, erlotinib, and dasatinib.

Table 1. Initial drug set used in the Therapeutically Guided Multidrug Optimization (TGMO) screen. Based on dose-response curves generated for each compound the $\mathrm{ED}_{20}$ dose was selected. Cell viability was measured using the CellTiter-Glo ${ }^{\circledR}$ luminescence assay following a 72-hour incubation with drugs.

\begin{tabular}{|c|c|c|c|}
\hline \multirow{2}{*}{ Drug } & \multicolumn{2}{|c|}{$786-\mathrm{O}$} & \multirow{2}{*}{ Target } \\
\hline & $\mathrm{ED}_{50}(\mu \mathrm{M})$ & $\mathrm{ED}_{20}(\mu \mathrm{M})$ & \\
\hline CI-994 & 11.5 & 1 & Class I HDACs \\
\hline LBH-589 & 0.01 & 0.002 & Class I and II HDACs \\
\hline SAHA & 3.7 & 1 & Class I and II HDACs \\
\hline tubacin & 6 & 2 & HDAC6 \\
\hline axitinib & 11.5 & 0.2 & VEGFRs, PDGFR \\
\hline erlotinib & 6.6 & 2 & EGFR \\
\hline BEZ-235 & 0.09 & 0.02 & mTOR \\
\hline dasatinib & 0.1 & 0.05 & SRC \\
\hline VX-680 & 33 & 0.65 & pan-Aurora Kinases \\
\hline sorafenib & 2.1 & 0.01 & VEGFRs, PDGFR \\
\hline
\end{tabular}


a
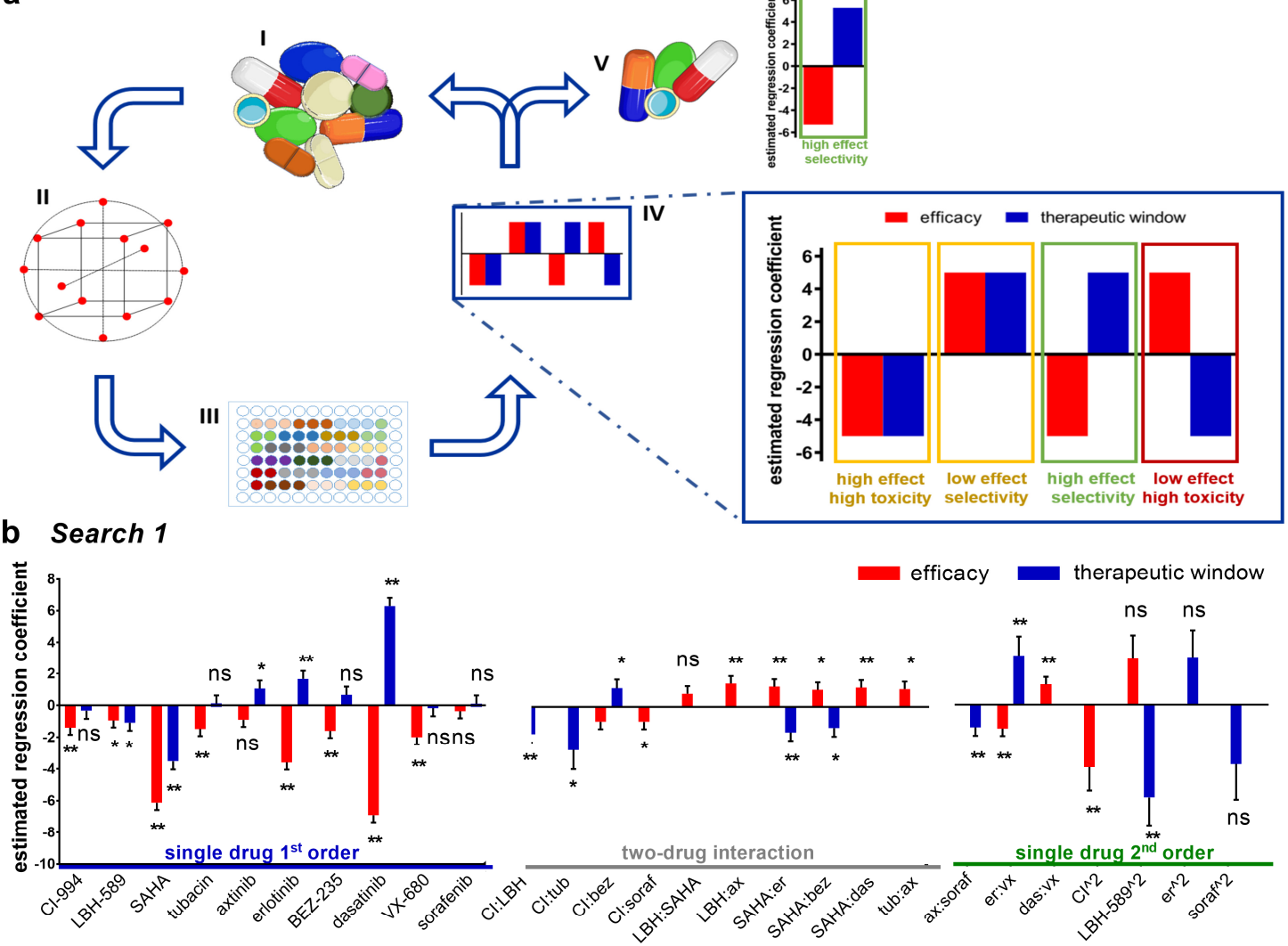

C Search 2
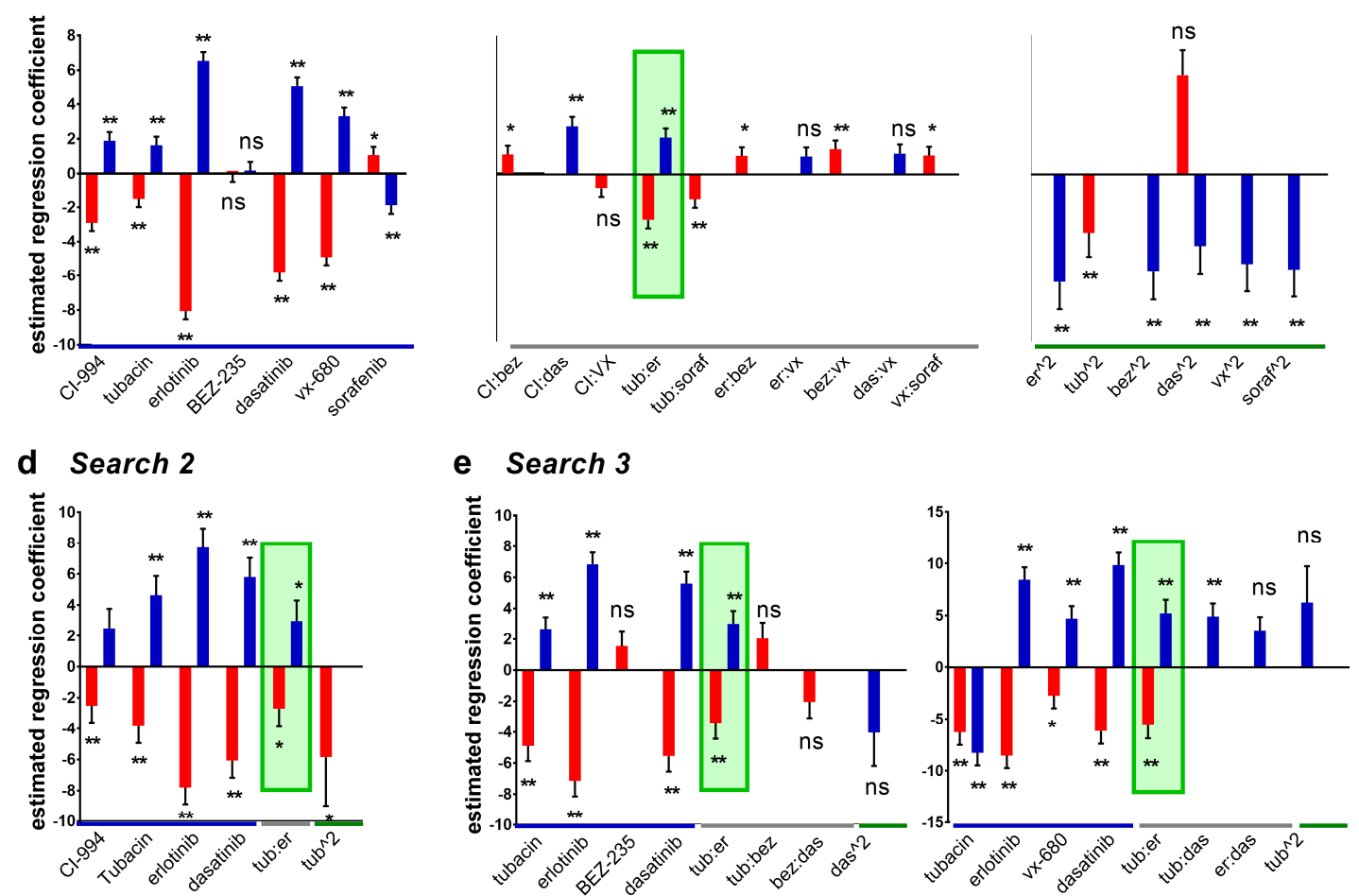

Figure 1. Therapeutically Guided Multidrug Optimization (TGMO)-based optimization of the drug combination. (a) Schematic overview of the TGMO-based optimization platform. (I) An initial set of ten drugs was selected. (II) Dose-response curves for each of ten drugs were generated for each cell type using inhibition of cell metabolic activity (ATP levels) as a measure for cell viability. The drug combination matrix to be tested was selected via an orthogonal array composite design (OACD). (III) The 
TGMO-based screen was initiated by experimentally testing a matrix of 91 drug combinations according to the drug combination matrix. (IV) The difference in drug efficacy between nonmalignant HEK-293T cells and malignant 786-O cells (in red) are defined as the therapeutic window (in blue). Regression coefficients of both the efficacy in 786-O cells and the therapeutic window were modeled using a step-wise second-order linear regression model. Orange frames indicate compounds showing not optimal drug interactions (either high efficacy with low toxicity or low efficacy with no toxicity), while compounds exhibiting an optimal activity profile (both effective and nontoxic) are framed in green. The red frame indicates compounds with poor activity profiles (lack of efficacy and considerable toxicity). (V) Data analysis allowed for drug selection, refinement of the searches and final the selection of the ODC. Estimated regression coefficients resulting from Search 1 (b), Search 2 (seven-drug and four-drug, $\mathbf{c}$ and d), and Search 3 (e). Regression coefficients corresponding to models of efficacy in 786-O cells are represented in red and the therapeutic window models are presented in blue. Green boxes highlight the most relevant synergistic activity consistent throughout the sequential searches and resulted in the selection of the optimal combination. Significance is represented with $*<0.05$ and ** $p<0.01$.

The efficacy of $\mathbf{C} \mathbf{1}$ was driven by a strong synergy between tubacin and erlotinib, as identified in the Searches 2 and 3 (Figure 1b-e, highlighted in green), as well as by the additive contribution of erlotinib and dasatinib. The activity of $\mathbf{C} \mathbf{1}$ showed highly selective and synergistic activity, as indicated by $\mathbf{C} 1$ outperforming the corresponding monotherapies $(p<0.01)$ and by the lack of activity in the nonmalignant HEK-293T cell line (Supplementary Figure S3a). Response surfaces generated from the regression model of data obtained in Search 2 (Figure 1e), demonstrated the synergistic interaction of tubacin and erlotinib (as evidenced by the slope of the surface), as well as the important contribution of all four compounds in the optimized combination (Supplementary Figure S3b).

In the final stage of the TGMO-based screen, Search 4, a broader dose range of these four compounds was considered and doses of compounds included in $\mathbf{C 1}$ were optimized. 786-O cell viability inhibition after incubation with drug combinations $\mathbf{C 2}-\mathbf{C} 5$ and corresponding monotherapies are presented in Figure 2a. C2 inhibited 786-O cell viability by $96 \%$, showing significantly greater activity than $\mathbf{C} \mathbf{1}(p<0.0071)$ and all single compound treatments. Drug combinations C1-C5 were only minimally active in HEK-293T, as well as normal human fibroblast NHDF $\alpha$ cells, confirming the successful application of the therapeutic window-based drug optimization. Moreover, C1-C5 also significantly outperformed the activity of non-optimal random drug combinations (Supplementary Figure S4), validating the TGMO-driven selection. The synergistic potential of each of the ODCs was further analyzed by calculating their respective Combination Indexes (CI) using Compusyn ${ }^{\circledR}$ software [19]. While CI values lower than one signify synergistic drug combinations (highlighted in green), CI higher than one indicates antagonism and a CI between these values indicates additivity (Figure 2a). C2 showed over 10-fold higher synergy $(\mathrm{CI}=0.04)$ than other ODCs and was hence selected for further evaluation.

The activity of $\mathbf{C} 2$ in cell viability inhibition was further tested in 3D homotypic (786-O cells) and 3D heterotypic (composed of 786-O cells, complemented with human NHDF $\alpha$ fibroblasts in ratio 1:1 and 10\% activated human endothelial cells, ECRF24) cell culture models (Figure 2b). C2 induced effective, approximately $80 \%$ cell viability inhibition in those models ( $p<0.01$ vs. CTRL and all monotherapies), confirming the results obtained in the 2D cell cultures (Figure 2a).

Since anti-angiogenic treatment may potentiate the overall treatment of cCRCC, we exposed human immortalized ECRF24 endothelial cells to C1-C5 and corresponding monotherapies. Interestingly, while $\mathbf{C} 2$ and $\mathbf{C} 3$ were virtually inactive in NHDF $\alpha$ cells, they were quite potent in activated human endothelial cells ECRF24 cells. These drug combinations induced inhibition ( $66 \%$ and $52 \%$, respectively) of ECRF24 cell viability. Treatment with C2 also led to increased apoptosis induction in endothelial cells $(12.6 \%$ vs. $1.6 \%$ in CTRL, Supplementary Figure S5a), which was mainly driven by tubacin, but did not influence endothelial cell migration in 2D (Supplementary Figure S5b) or 3D network formation (Supplementary Figure S5c). The anti-angiogenic potential of $\mathbf{C} 2$ was further confirmed in the in vivo developmental chorioallantoic membrane (CAM) model [20,21], where $\mathbf{C} 2$ significantly 
$(p<0.0001)$ reduced the development of the microvasculature (quantified by the number of branching points $/ \mathrm{mm}^{2}$, Figure 2c). These results suggest therefore a potential dual, i.e., anticancer and angiostatic, mechanism of action of $\mathbf{C} 2$.

a

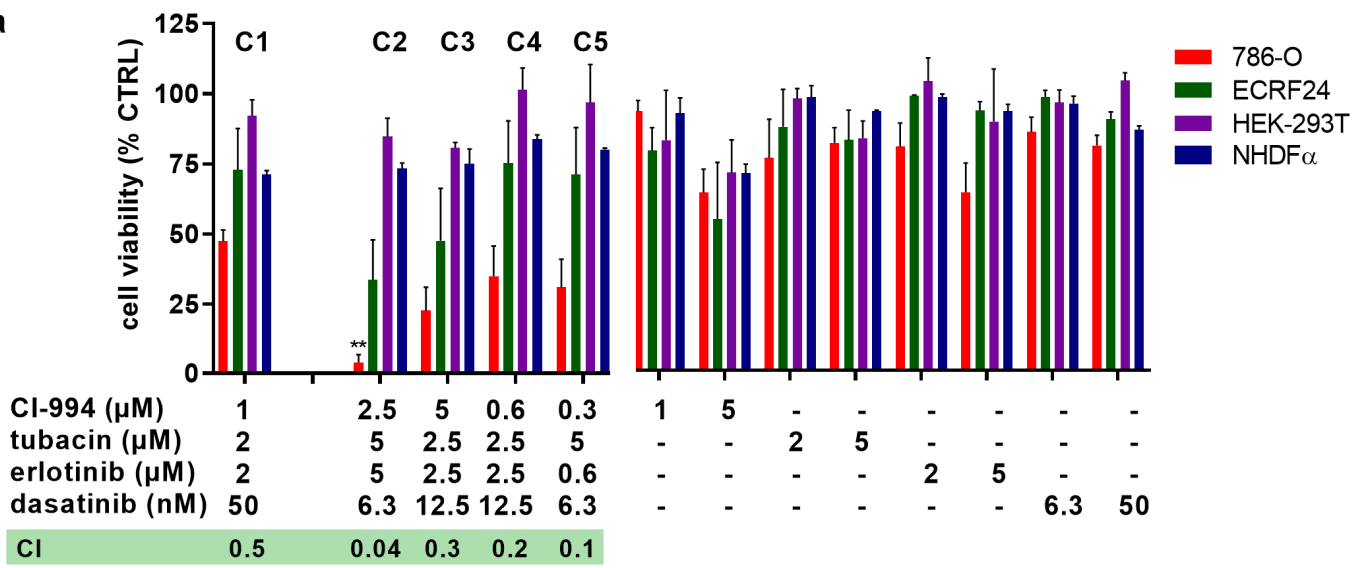

b
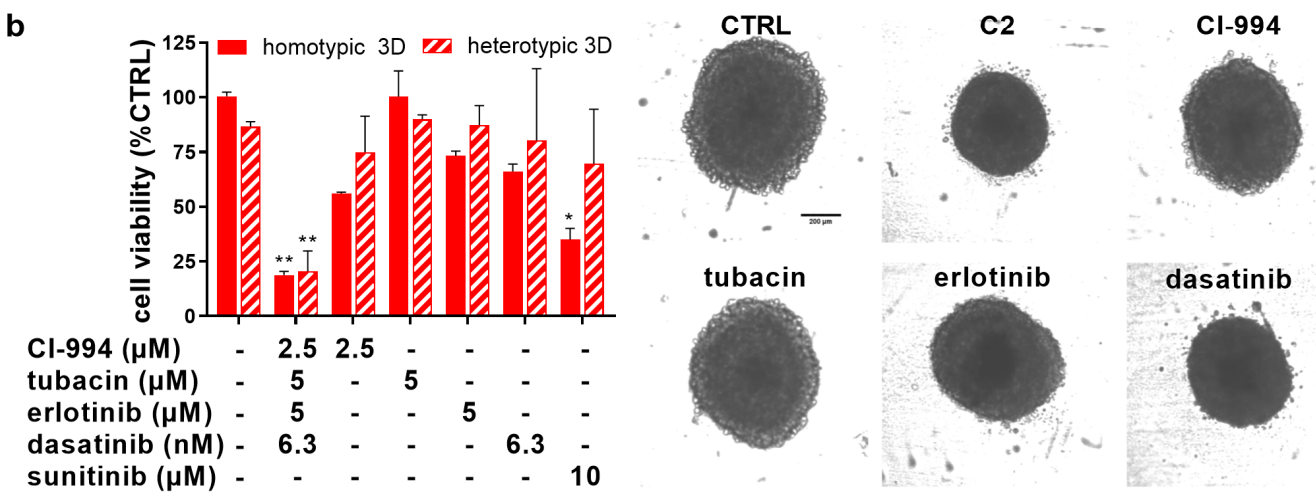

C
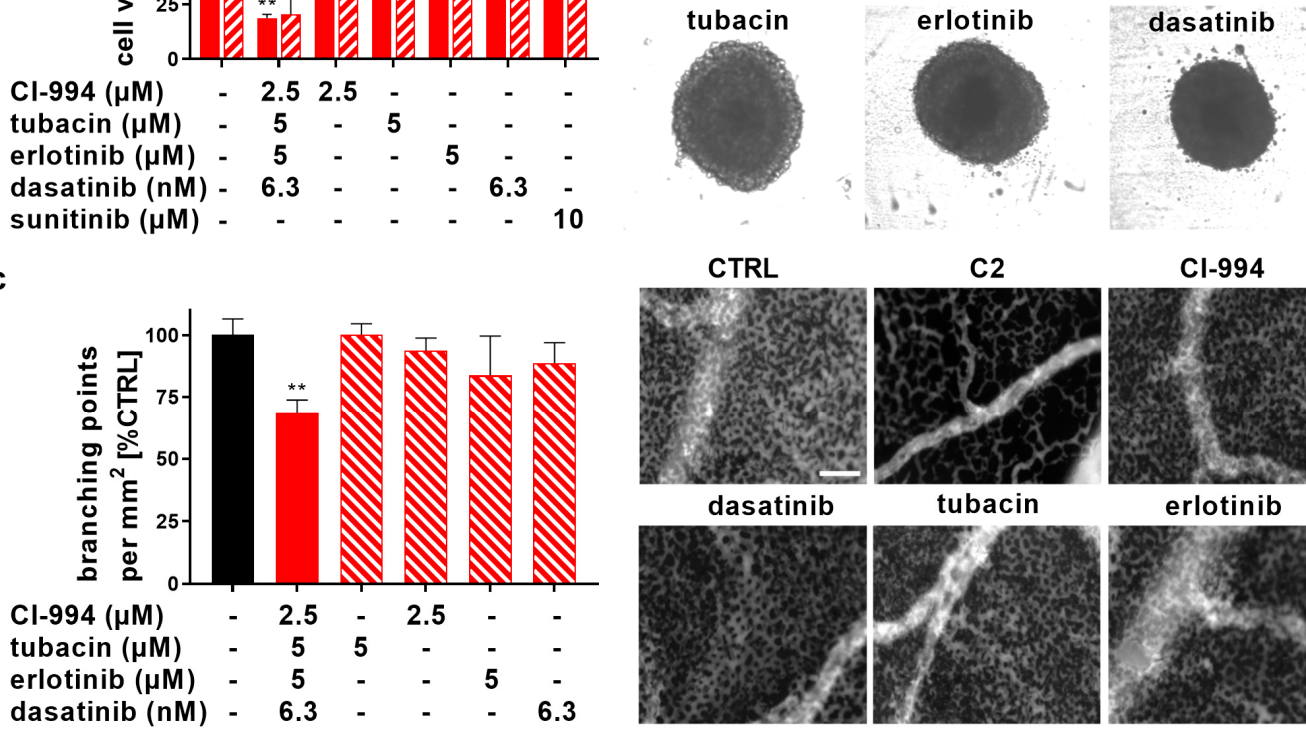

Cl-994

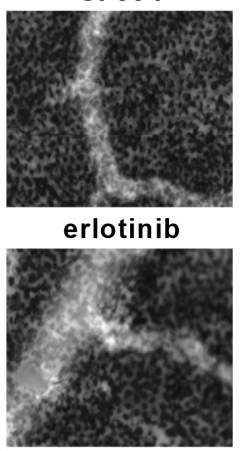

Figure 2. Dose optimization and validation of the OCD efficacy in 3D cell cultures with sunitinib-resistant cells and anti-angiogenic ODC potential in the chorioallantoic membrane model (CAM). (a) The efficacy of the five most promising drug combinations (C1-C5) derived from the dose optimization with C1, identifying $\mathbf{C} 2$ as the most effective drug combination. Corresponding single drug treatments are presented for the 786-O cell line, non-malignant renal HEK-293T control cells, as well as in nonmalignant $\mathrm{NHDF} \alpha$ fibroblasts and activated ECRF24 endothelial cells. Green box: the "combination index" (CI) values for each drug combination with $\mathrm{CI}<1$ indicating synergy (highlighted in green), 0 and CI $>1$ indicating antagonism. ${ }^{*} p<0.05$ and ${ }^{* *} p<0.01$ represent significant increased activity of $\mathbf{C} 1$ compared to $\mathbf{C 2}-\mathbf{C} 5$ and corresponding single drug treatments as determined by a one-way ANOVA with post hoc Sidak's multiple comparison test from $\mathrm{N}=2-4$ independent experiments. (b) Efficacy and representative 
images of the dose-optimized drug combination C2 in 3D homotypic (786-O) spheroids or in 3D coculture heterotypic spheroids containing human fibroblasts, 786-O (1:1) and 10\% ECRF24 endothelial cells. Sunitinib at $10 \mu \mathrm{M}$ was used as a positive control. Scale bar represents $200 \mu \mathrm{m}$ for all images. (c) In vivo inhibition developmental angiogenesis evaluated in the chorioallantoic membrane (CAM) model of the chicken embryo following two consecutive days of topical drugs administration. Fluorescence angiograms show the inhibition of capillary growth in CAM treated with C2 as presented by the quantification of the number of branching points $/ \mathrm{mm}^{3}$ based on the automated image-analysis. ** $p<0.01$ represents significance versus CTRL as determined by a one-way ANOVA with post hoc Sidak's multiple comparison test from $\mathrm{N}=2$ independent experiments $(\mathrm{n}=4-15)$. Error bars represent \pm SEM. Scale bar represents $800 \mu \mathrm{m}$.

\subsection{C2 Activity on Cell Cycle Regulation, Actin Cytoskeleton Reorganization, and Nuclear Structures}

In order to investigate the potential mechanism of action of $\mathbf{C 2}$, we monitored cell cycle distribution and the frequency of apoptosis with propidium iodide staining using flow cytometry. Exposure of 786-O cells to C2 for $24 \mathrm{~h}$ resulted in higher apoptosis levels $(8.1 \%$ vs. $1.1 \%$ in CTRL, $p=0.0109$, Supplementary Figure S6a, top graph). After $72 \mathrm{~h}$ of drug incubation, the higher incidence of apoptosis was no longer observed, however, a G2/M phase arrest was registered (12.3\% and 21.3\% for CTRL and C2, respectively, $p=0.0083$, Supplementary Figure S6a bottom graph). Sunitinib at a concentration of $10 \mu \mathrm{M}$ was used as positive control.

Morphological changes in C2 treated cells were also investigated based on fluorescent phalloidin (f-actin marker) and nuclear DAPI (4',6-diamidino-2-phenylindole) staining. After C2 treatment, we observed changes in the actin cytoskeleton morphology. The cells presented enhanced stress fibers in the center, but also the formation of $\mathrm{f}$-actin cables running along the cell borders (Supplementary Figure S6c, white arrows). Nevertheless, C2 did not affect 786-O migration in a 2D migration assay (Supplementary Figure S7). DAPI staining also indicated that C2 treatment led to a three-fold increase $(7.5 \% \pm 1.3 \%)$ in the number of micronuclei vs. CTRL treatment $(1.8 \pm 0.3 \%, p<0.001$, Supplementary Figure S6b,c, red arrow). Micronuclei induction is a marker for chromosome segregation errors or chromosome aberrations, known to be linked to genomic instability and chromosomal damage [22]. The same treatment also induced other significant nuclear abnormalities (Supplementary Figure S6c, yellow arrow), mostly driven by CI-994. The nuclear abnormalities and the G2/M enrichment in the FACS profile suggested potential defects in cell division, such as chromosome segregation errors (micronuclei) or cytokinesis failure (multi-nuclei) [23].

\subsection{C2 Prevents the Clustering of Multipolar Spindles in 786-O Cells}

To test whether $\mathbf{C} 2$ treatment affects cell division, we monitored cell proliferation by live-cell imaging. 786-O cells were treated with $0.1 \%$ DMSO in the cell culture medium (CTRL) or C2 and stained with the live-cell dye SiR-tubulin (microtubule marker) to label the mitotic spindle, monitor mitotic progression, and detect any cytokinesis failure. All experiments were performed in the presence of Valspodar $(4 \mu \mathrm{M})$, a multidrug pump inhibitor that prevented SiR-tubulin removal from the cell (this concentration of Valspodar had no effect on the efficacy of C2). Live cells were recorded for $24 \mathrm{~h}$ at a temporal resolution of $3 \mathrm{~min}$. We found that during these $24 \mathrm{~h}$, fewer $\mathrm{C} 2$ treated cells entered mitosis compared to CTRL ( $35 \%$ vs. $66 \%$ ) and more of them died in interphase before any mitotic entry ( $6 \%$ vs. $1 \%$; Figure 3a), pointing to a cell cycle delay. Furthermore, C2-treated cells exited mitosis later, as the mean time between nuclear envelope breakdown (NEBD, time point at which the spindle is set up) and the appearance of the cleavage furrow increased from $30 \pm 10 \mathrm{~min}$ in CTRL-treated cells to $42 \pm 6$ min (Figure 3b,c). Importantly, a significantly higher number of C2-treated cells died during mitosis ( $3 \%$ vs. $0 \%$ in CTRL) or after mitotic exit in the ensuing interphase $(12 \%$ vs. $0.5 \%$ in CTRL, Figure 3d). While our analysis did not reveal any cytokinesis defect, we frequently found multipolar spindles in C2 treated cells (44\% vs. 18\% in CTRL-treated cells, Figure 3e). 
a

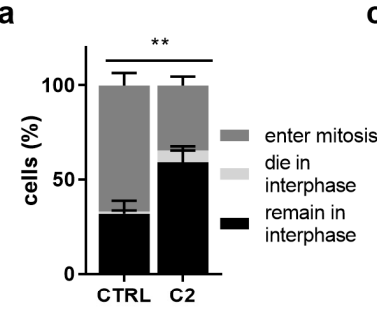

b

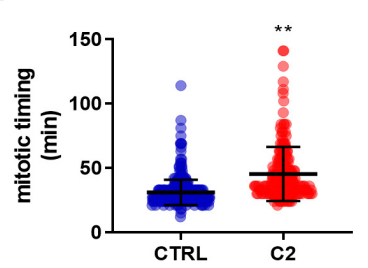

d

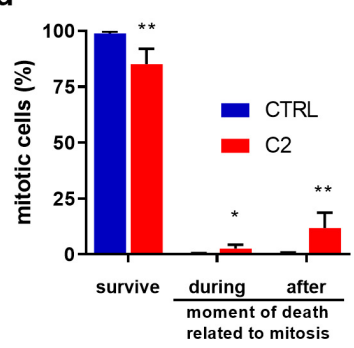

h
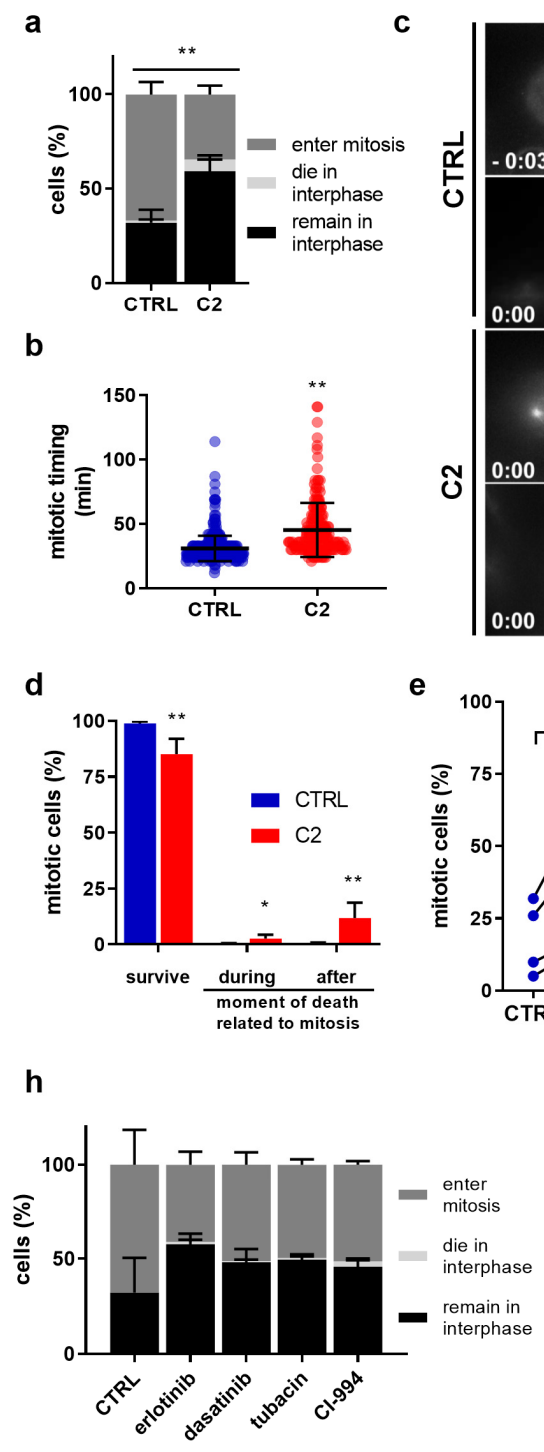
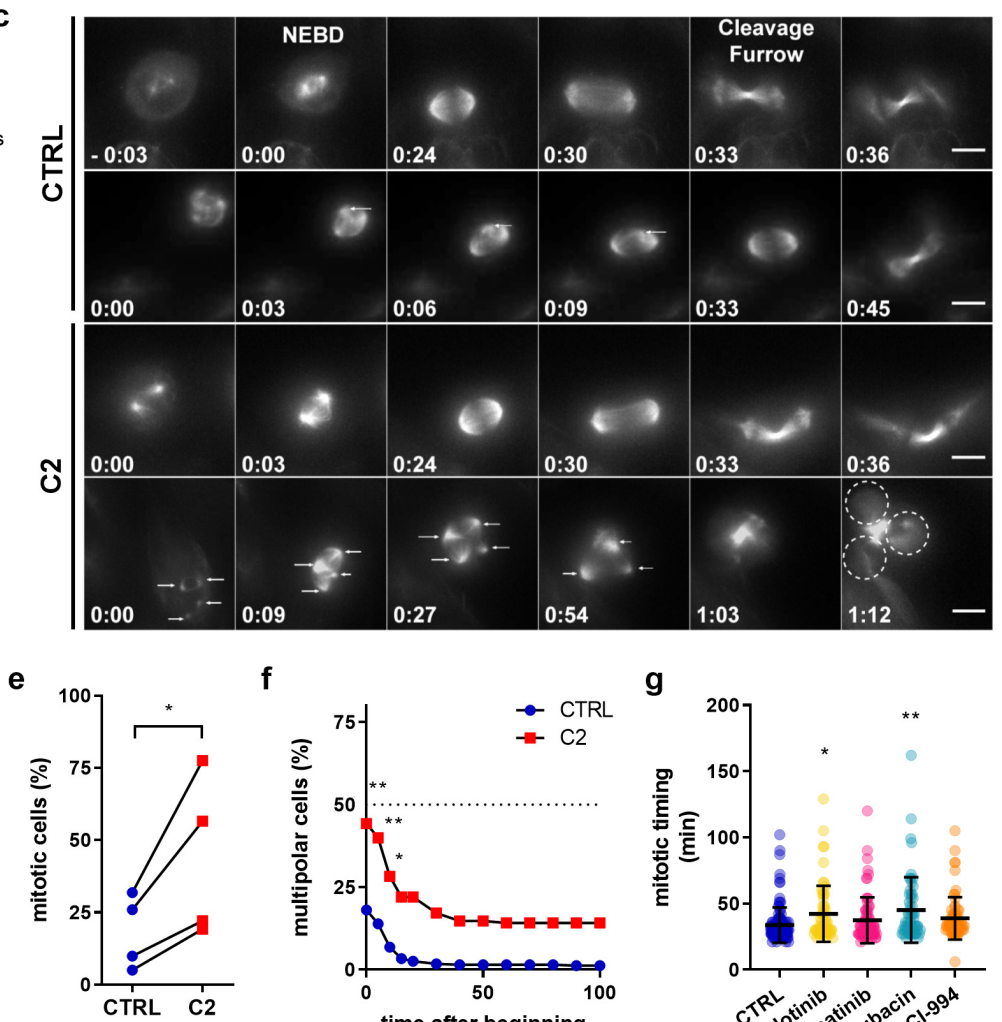

f
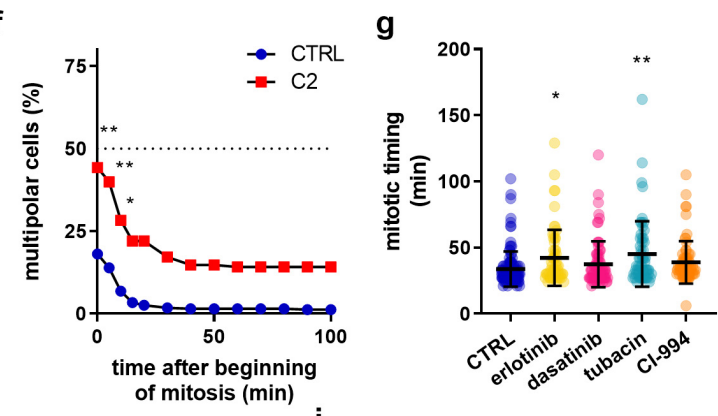

i

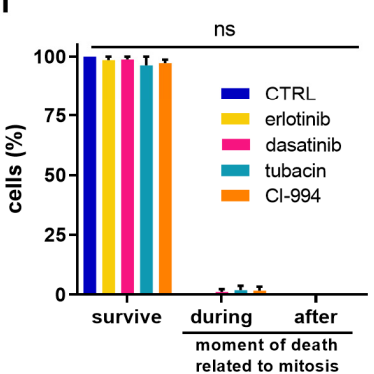

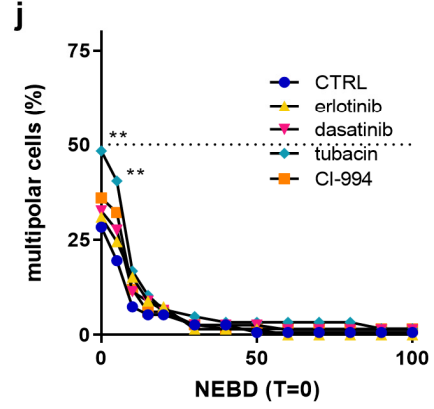

Figure 3. C2 prevents centrosome clustering in 786-O cells during metaphase. (a) Outcomes of 786-O cells treated with CTRL (0.1\% DMSO) or C2 during 24 h movies. ${ }^{* *} p<0.0001$ vs. CTRL in a Fisher's exact test, $\mathrm{N}=4$ experiments with $\mathrm{n}=606$ (CTRL) and $\mathrm{n}=603$ (C2) cells (b) Mitotic timing is defined as the time between nuclear envelope breakdown (NEBD) and cleavage furrow formation. ${ }^{* *} p<0.001$ vs. CTRL in a Mann-Whitney Test from $N=4$ independent experiments with a total of $n=206-406$ cells. (c) Time-lapse images of CTRL (0.1\% DMSO) or C2-treated 786-O cells undergoing mitosis, stained with SiR-Tubulin. Mitotic timing in h:mins using nuclear envelope breakdown (NEBD) as $\mathrm{T}=0$. The arrows in the C2-treated multipolar cells indicate spindle poles, dashed circles the daughter cells after mitotic completion. Scale bar $=10 \mu \mathrm{m}$. (d) Mitotic outcomes of 786-O cells treated with CTRL ( $0.1 \%$ DMSO) or C2. ${ }^{* *} p<0.0001$ and ${ }^{*} p<0.05$ vs. CTRL in a Fisher's exact test, $\mathrm{N}=4$ independent experiments with $\mathrm{n}=406$ (CTRL) and $\mathrm{n}=206$ (C2) cells. (e) Percentage of multipolar cells in individual experiments. * $p<0.05$ vs. CTRL in paired two-tailed T-test from $\mathrm{N}=4$ independent experiments; $\mathrm{n}=406$ (CTRL) and 206 (C2) cells. (f) Percentage of multipolar cells over time. ${ }^{* *} p<0.005$ and ${ }^{*} p<0.05$ for the first three points compared to CTRL in a two-way ANOVA with Sidak's multiple comparisons test from $\mathrm{N}=4$ independent experiments with $\mathrm{n}=406$ (CTRL) and $\mathrm{n}=206$ (C2) cells. (g) Mitotic timing of 786-O cells treated with the single drugs used for C2. Significances of ${ }^{* *} p=0.0008$ and ${ }^{*} p=0.0236 \mathrm{vs}$. CTRL 
group in a two-way ANOVA with Tukey's multiple comparisons test from $\mathrm{N}=4$ experiments with $\mathrm{n}=62-123$ cells. (h) Outcomes of 786-O cells treated with CTRL or monotherapies during $24 \mathrm{~h}$ movies. $\mathrm{N}=4$ experiments with $\mathrm{n}=123-160$ cells. (i) Mitotic outcome of $786-\mathrm{O}$ cells treated with CTRL $(0.1 \%$ DMSO) or monotherapies. None of the monotherapies was significantly different compared to CTRL group in a two-way ANOVA with Tukey's multiple comparisons test from $\mathrm{N}=4$ experiments with $\mathrm{n}=62-123$ cells. (j) Percentage of multipolar cells over time. ${ }^{* *} p<0.01$ for the first two points of tubacin treated cells vs. CTRL in a two-way ANOVA with Sidak's multiple comparisons test from $\mathrm{N}=4$ experiments with $\mathrm{n}=62-123$ cells. Error bars represent SEM for all graphs.

Such multipolar spindles can arise in the presence of extra centrosomes, the main microtubule organizing centers in mitosis [24], or can be caused by fragmentation of the pericentriolar material (PCM). Persistent multipolar spindles result in multipolar cell divisions which in turn frequently leads to cell death in the ensuing interphase due to massive chromosome mis-segregations $[25,26]$. Cancer cells possess a survival mechanism to overcome this problem called spindle pole or centrosome clustering, where supplementary spindle poles are gathered together to form a pseudo-bipolar spindle, a phenomenon that was also visible in 786-O cells (Figure 3c, CTRL condition, white arrows). We, therefore, plotted the percentage of multipolar spindles over time and found that $\mathbf{C} 2$ treated cells were severely delayed in their ability to cluster multipolar spindles compared to the CTRL, resulting in 15\% of the cells failing to resolve their multipolar spindles over the entire course of mitosis (Figure 3c,f). Importantly, these mitotic phenotypes were specific for the C2-treatment, as apart from a small mitotic delay in cells treated with erlotinib or tubacin, no equivalent phenotype could be observed in cells treated with the single components of the $\mathbf{C} 2$ combination (Figure $3 \mathrm{~g}-\mathrm{j}$ ).

As an additional control, we also tested the effects of C2 on HEK-293T cell division, as this cell line had not shown a large decrease in viability after $\mathbf{C} 2$ treatment (Figure 2a). Live cell imaging performed on HEK-293T cells showed that even though C2 prolonged mitotic timing and reduced the number of cells entering mitosis (Supplementary Figure S8a-c), it did not increase the proportion of cells with multipolar spindles, unlike what has been observed in 786-O cells (Supplementary Figure S8d and Figure 3f). Moreover, no major cell death was observed in interphase (1.5\% vs. $0 \%$ in CTRL), during mitosis ( $2 \%$ vs. $0 \%$ in CTRL) or after mitotic exit in the ensuing interphase $(1 \%$ vs. $0 \%$ in CTRL) (Supplementary Figure S8c,e). This suggested that C2 specifically induces cell death in 786-O by prolonging multipolarity during mitosis.

\subsection{C2 Activity Prevents Spindle Pole Clustering in Sunitinib-Resistant 786-O Cells}

The first-line therapy for advanced ccRCC is sunitinib, a tyrosine kinase inhibitor to which patients rapidly develop resistance. Therefore, we evaluated whether our optimized drug combination $\mathbf{C 2}$ would also be effective in sunitinib-resistant cells (786-OsunR). First, 786-O cells were chronically exposed to sunitinib treatment $(1 \mu \mathrm{M})$ and dose-response curves were generated to validate resistance induction (Supplementary Figure S9a). Moreover, intracellular and intralysosomal accumulation, one of the resistance mechanism of sunitinib [27], was observed (Supplementary Figure S9b). C2 effectively inhibited the viability of 786-OsunR cells and significantly outperformed all single drug treatments (Figure $4 \mathrm{a}, p<0.01$ vs. CTRL and all monotherapies).

To determine whether $\mathbf{C} 2$ also impaired cell division in 786-OsunR cells, we performed live cell-imaging experiments after CTRL or C2 treatment. While C2 does not affect mitotic timing (Figure $4 \mathrm{~b}$ ), it prevented spindle pole clustering into a bipolar spindle over the course of mitosis in the 786-OsunR cells ( $30 \%$ vs. 5\% for CTRL; Figure 4c,d). Moreover, C2 treatment led to a higher incidence of cell death during mitosis ( $17 \%$ vs. $1 \%$ in CTRL) and after mitotic exit $(41 \%$ vs. $2 \%$ in CTRL; Figure 4e). We conclude that C2 prevented bipolar spindle formation in a large segment of the 786-OsunR cells and led to mitotic cell death independent of sunitinib-induced resistance [28]. Nevertheless, we note that $\mathbf{C} 2$ treatment led to a high proportion of cell death in interphase before cells could enter mitosis, implying that $\mathbf{C} 2$ also has a mitosis-independent mechanism of action in the 786-OsunR cells (Figure 4f). 
Multipolar spindles can arise via different mechanisms: fragmentation of the pericentriolar material, premature splitting of the two centrioles within the centrosomes, or presence of more than two centrosomes, as is frequently the case in cancer cells [29]. 786-O cells have been reported to only have a low percentage of cells with more than two centrosomes [30]. We therefore investigated the nature of the multipolar spindles in 786-O and 786-OsunR cells by immunofluorescence. Cells were synchronized in mitosis with the microtubule-depolymerizing drug nocodazole, released from this treatment to allow them to form a spindle and stained for $\alpha$-tubulin (spindle marker), $\gamma$-tubulin (pericentriolar material marker), and centrin (centriole marker). Our analysis revealed that most multipolar spindles in both cell lines treated with or without $\mathbf{C} 2$ resulted from the fragmentation of the pericentriolar material (Figure 4g,h).
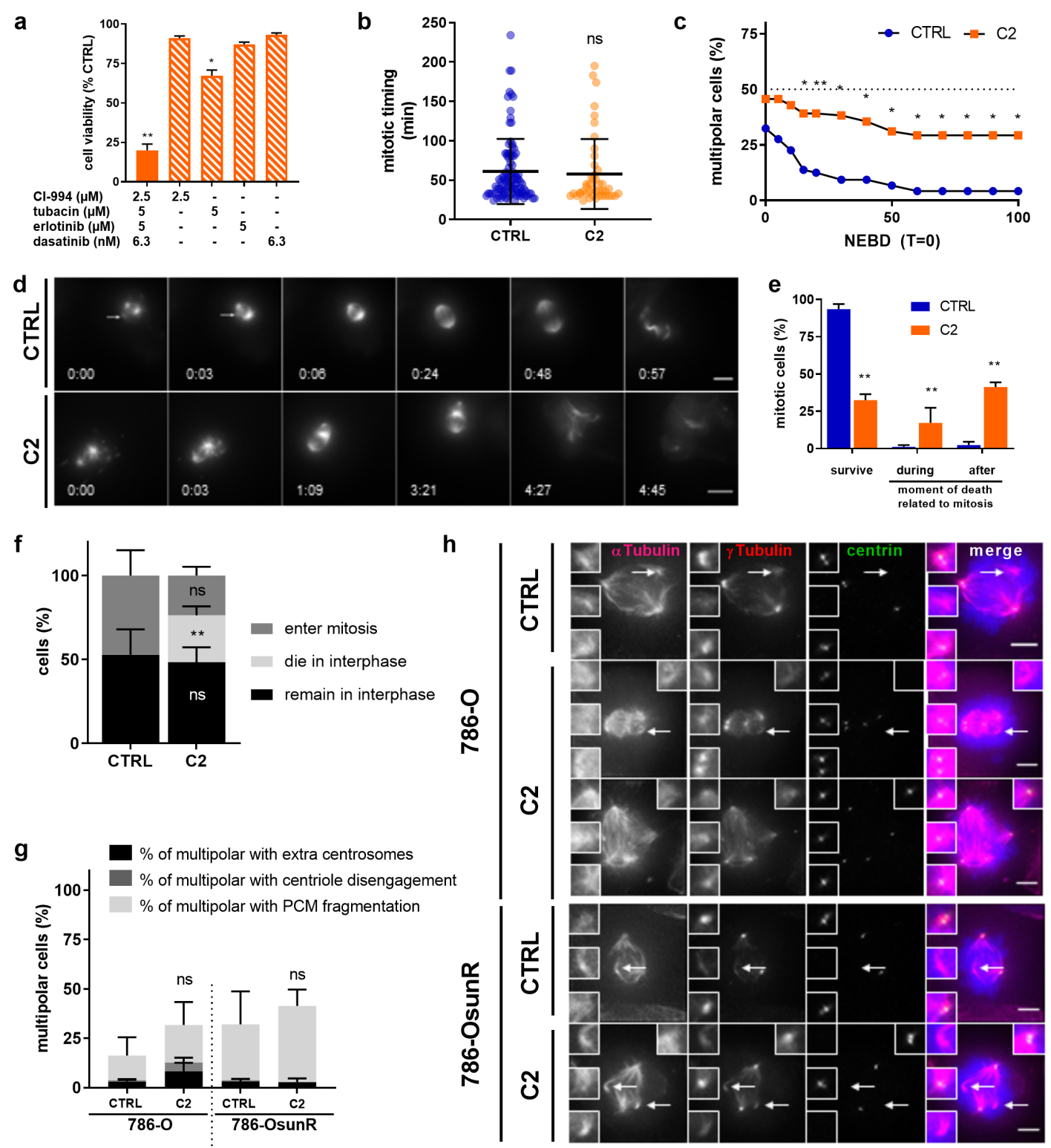

Figure 4. C2 activity also prevents spindle pole clustering in sunitinib-resistant 786-O cells. (a) Efficacy of $\mathbf{C} 2$ and corresponding single drug treatments in 786-O cells chronically exposed to sunitinib treatment (786-OsunR). ${ }^{* *} p<0.0001$ and ${ }^{*} p<0.05$ represent significances versus all corresponding single drug treatments from $\mathrm{N}=5$ independent experiments as determined by a one-way ANOVA with post-hoc Tukey's multiple comparison test. (b) Mitotic timing of 786-OsunR treated with CTRL ( $0.1 \%$ DMSO) or C2. $p=0.2262$ compared to CTRL as determined by a Mann-Whitney Test from $\mathrm{N}=3$ experiments with $n=65-106$ cells. (c) Percentage of multipolar cells over time. ${ }^{*} p<0.05$ and ${ }^{* *} p<0.005$ vs. CTRL 
group in a two-way ANOVA with Sidak's multiple comparisons test from $\mathrm{N}=3$ experiments with $\mathrm{n}=$ 65-106 cells for CTRL and C2-treated cells. (d) Time-lapse images of CTRL and C2-treated 786-OsunR cells stained with SiR-Tubulin. Mitotic timing in h:mins using NEBD as T $=0$. Arrows in the CTRL multipolar 786O-sunR cells indicate spindle poles. Scale bar $=10 \mu \mathrm{m}(\mathbf{e})$ Mitotic outcome of 786-O-sunR cells. ${ }^{* *} p<0.0001$ vs. CTRL in a Fisher's exact test, $\mathrm{N}=3$ experiments with $\mathrm{n}=106-65$ cells for CTRL and C2-treated cells. (f) Outcomes of 786-OsunR cells treated with CTRL or C2 during the $24 \mathrm{~h}$ movie. ${ }^{* *} p<0.0001$ vs. CTRL in Fisher's exact test, $N=3$ experiments with $n=221$ and $n=259$ cells (g) Percentage of fixed multipolar cells with extra centrosomes, disengaged centrioles or fragmented pericentriolar material (PCM) after $12 \mathrm{~h}$ of CTRL or C2 treatment. Within each cell line, C2-treated cells were compared to CTRL cells in Fisher's exact test from $\mathrm{N}=3$ experiments with $\mathrm{n}=112-164$ cells. (h) Representative immunofluorescence images of 786-O and 786-OsunR cells, treated for $12 \mathrm{~h}$ with CTRL or C2, stained for $\alpha$-tubulin (magenta), $\gamma$-tubulin (red), centrin (green), and DAPI (blue). Scale bar $=5 \mu \mathrm{m}$. White arrows indicate poles formed by PCM fragmentation. Error bars represent SEM in all graphs.

\subsection{C2 Treatment Shows Greater Efficacy in the Cells with Abnormal Centrosome Numbers}

Based on our results, we hypothesized that one of the main mechanisms by which $\mathrm{C} 2$ generally kills cancer cells is by preventing spindle pole clustering. To strengthen this hypothesis we tested whether $\mathbf{C} 2$ can also target cancer cells from other tissues displaying elevated centrosome/centriole numbers, which should be prone to form multipolar spindles. We evaluated its effects on two melanoma cell lines that have been reported to either have low (M14) or abnormally high (MDA-MB-435) levels of centrosome/centriole numbers [30]. Consistent with these reports, we found that 65\% of MDA-MB-435 had more than four centrioles (vs. 15\% in M14 cells; Figure 5a,b). Interestingly, C2 inhibited cell viability much more efficiently in MDA-MB-435 than in M14 cells (90 $\pm 10 \%$, vs. $40 \pm 11 \%$; Figure 5c). Live-cell imaging indicated that the $\mathbf{C} 2$ treatment resulted in fewer cells entering mitosis and a mitotic delay for both cell lines (Figure 5d,e), matching our results in 786-O, 786-OsunR, and HEK-293T cells. During mitosis, more MDA-MB-435 cells failed to divide properly and died in mitosis after C2 treatment compared to M14 cells (Figure 5f). Moreover, C2 treatment resulted in an elevated number of persistent multipolar spindles in MDA-MB-435 cells, but not in M14 cells (Figure 5g,h).
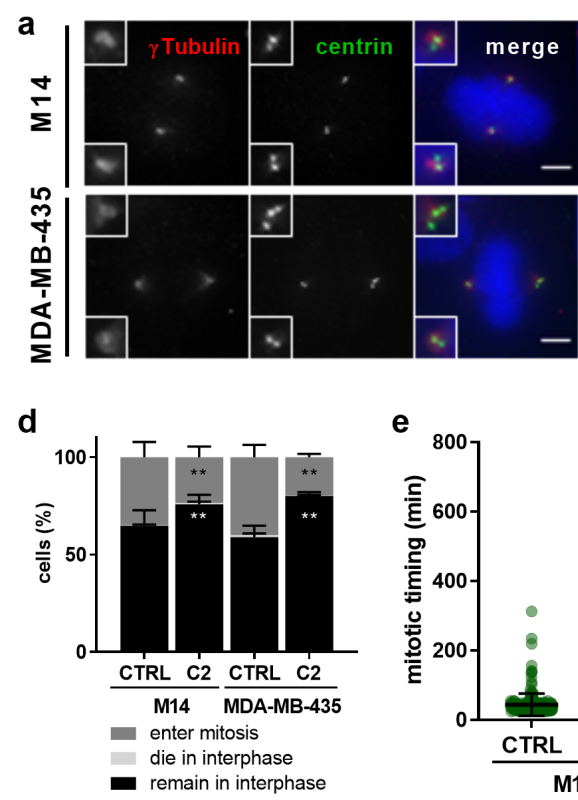

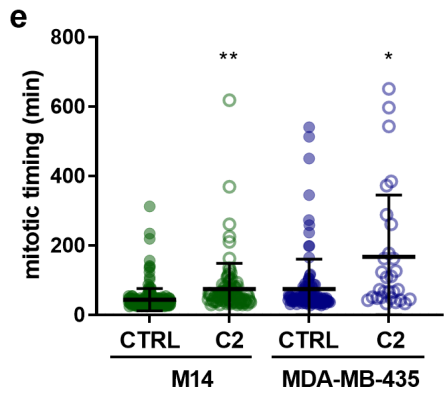

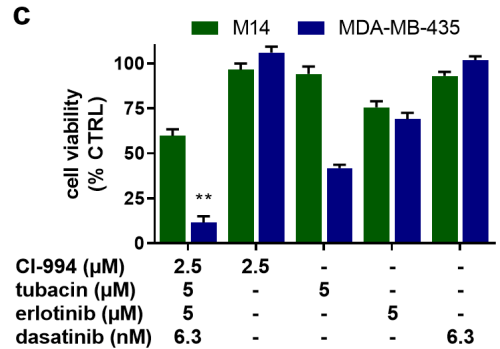

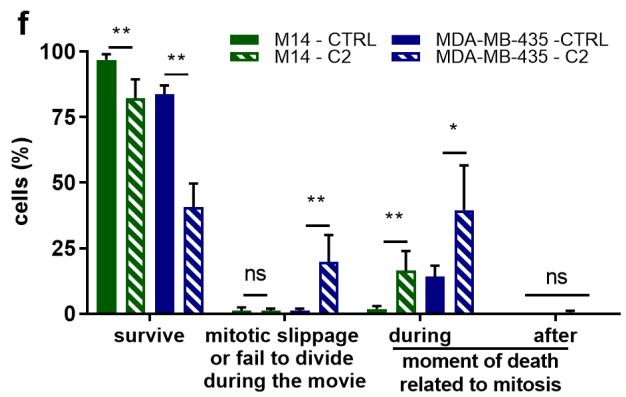

Figure 5. Cont. 

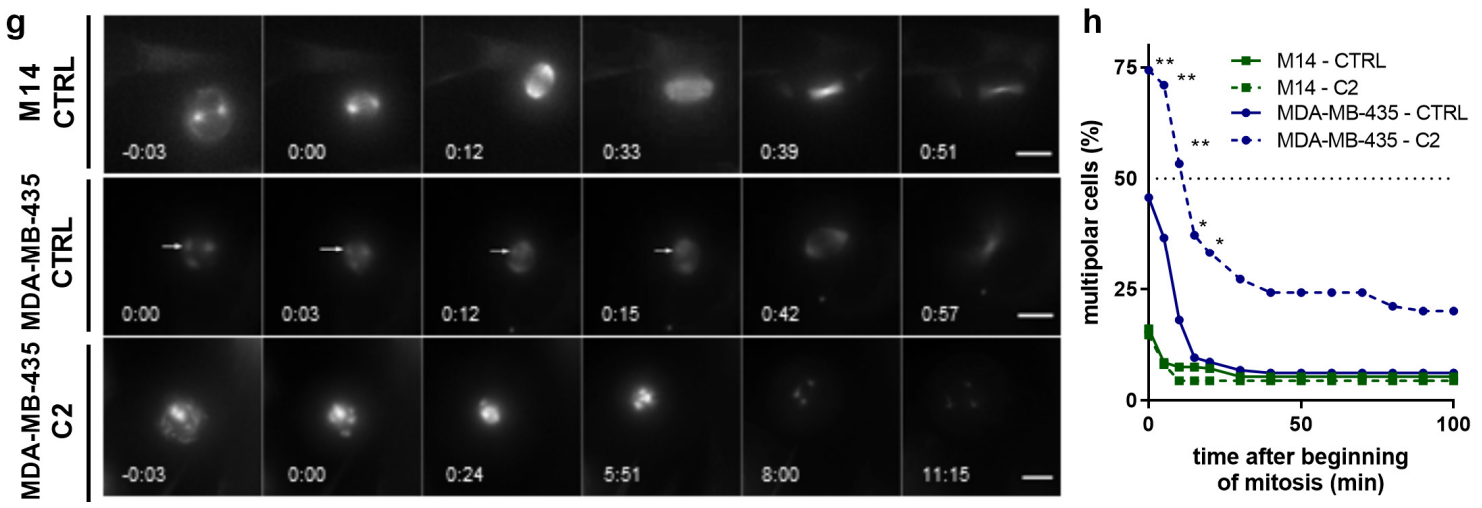

Figure 5. Effects of $\mathbf{C} 2$ treatment on melanoma cells with normal or elevated number of centrosomes. (a) Representative images of M14 and MDA-MB-435 cells stained for centrin (green), $\gamma$-tubulin (red), and DAPI (blue). Scale bar $=5 \mu \mathrm{m}$. (b) Image-based quantification of the number of centrioles per cells. Results from $\mathrm{N}=4$ experiments with $\mathrm{n}=165$ cells for M14 and 164 cells for MDA-MB-435 cells. (c) Efficacy of C2 and corresponding single drug treatments in M14 and MDA-MB-435 cells, ${ }^{* *} p<0.01$ versus all corresponding single drug treatments in a one-way ANOVA with posthoc Tukey's multiple comparison test. (d) Outcomes of M14 and MDA-MB-435 cells treated with CTRL (0.1\% DMSO) or C2 during 24 h movies. ${ }^{* *} p<0.0001$ vs. CTRL in a Fisher's exact test, $\mathrm{N}=3$ experiments, $\mathrm{n}=610-515$ cells for M14, and n=355-297 cells for MDA-MB-435 cells. (e) Mitotic timing from NEBD until formation of a cleavage furrow in min. ${ }^{* *} p<0.0001$ for M14 cells and $* p=0.0002$ for MDA-MB-435 cells, (CTRL vs. C2) in a Mann-Whitney Test from $\mathrm{N}=3$ experiments with $\mathrm{n}=227-122$ cells for M14 and $\mathrm{n}=140-60$ cells for MDA-MB-435. (f) Mitotic outcome of M14 and MDA-MB-435 cells. ${ }^{* *} p<0.0001$ and ${ }^{*} p<0.001$ vs. CTRL in a Fisher's exact test, $\mathrm{N}=3$ experiments with $\mathrm{n}=227-122$ cells for M14 and $\mathrm{n}=140-60$ cells for MDA-MB-435. (g) Time-lapse images of M14 and MDA-MB-435 cells undergoing mitosis, stained with SiR-Tubulin. Mitotic timing in h:mins using nuclear envelope breakdown (NEBD) as T $=0$. Arrows indicate spindle poles in the C2-treated multipolar MDA-MB-435 cells. Scale bar $=10 \mu \mathrm{m}$. (h) Percentage of multipolar cells over time. ${ }^{*} p<0.05$ and ${ }^{* *} p<0.01$ vs. CTRL in a two-way ANOVA with Sidak's multiple comparisons test from $N=3$ experiments with $n=227-122$ (CTRL) and $n=140-60$ cells (C2) M14 and MDA-MB-435 cells. Error bars represent SEM in all graphs.

These results were consistent with our hypothesis and implied that the efficacy of the C2-treatment is linked to the propensity of cancer cell lines to form multipolar spindles due to abnormal centrosome/centriole numbers (MDA-MB-435) or fragmentation of the pericentriolar material (786-O); see schematic model in Figure 6. 
a. normal mitosis

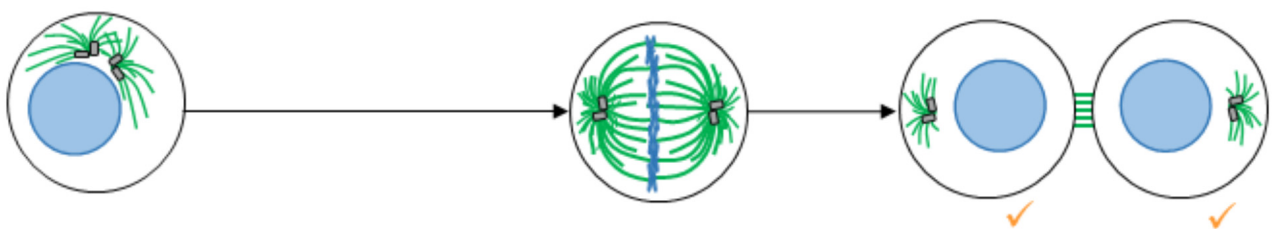

b. centriole splitting or PCM fragmentation

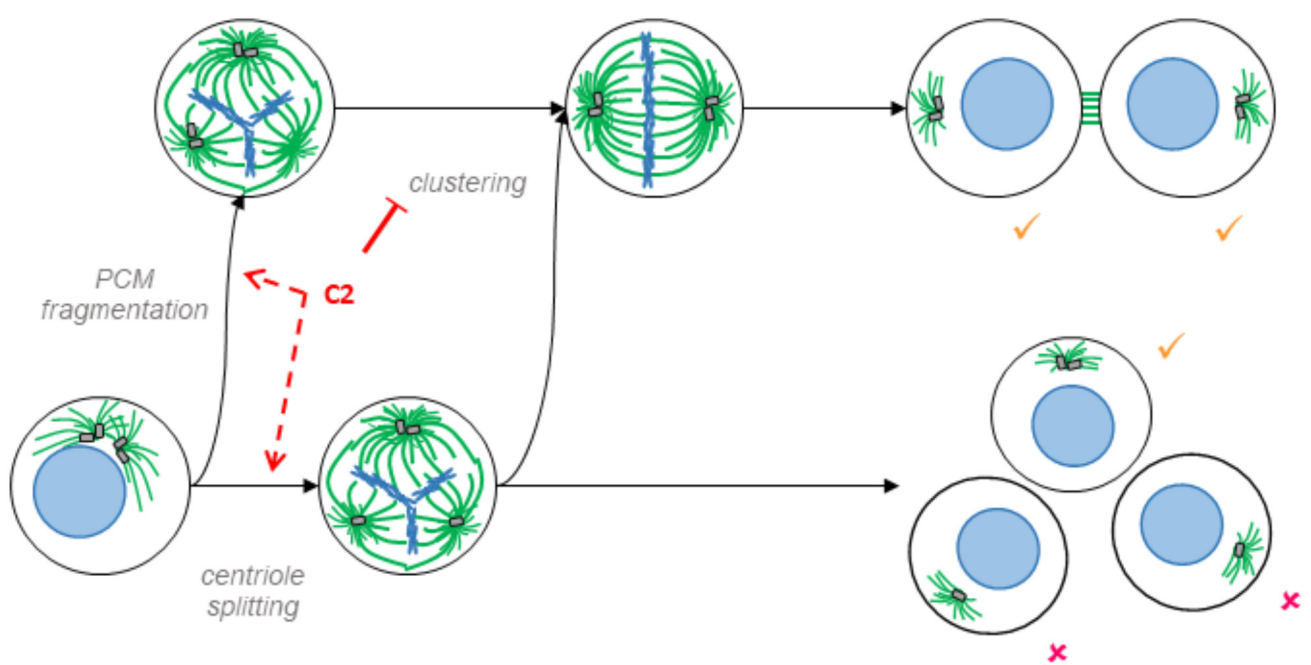

c. supernumerary centrosomes

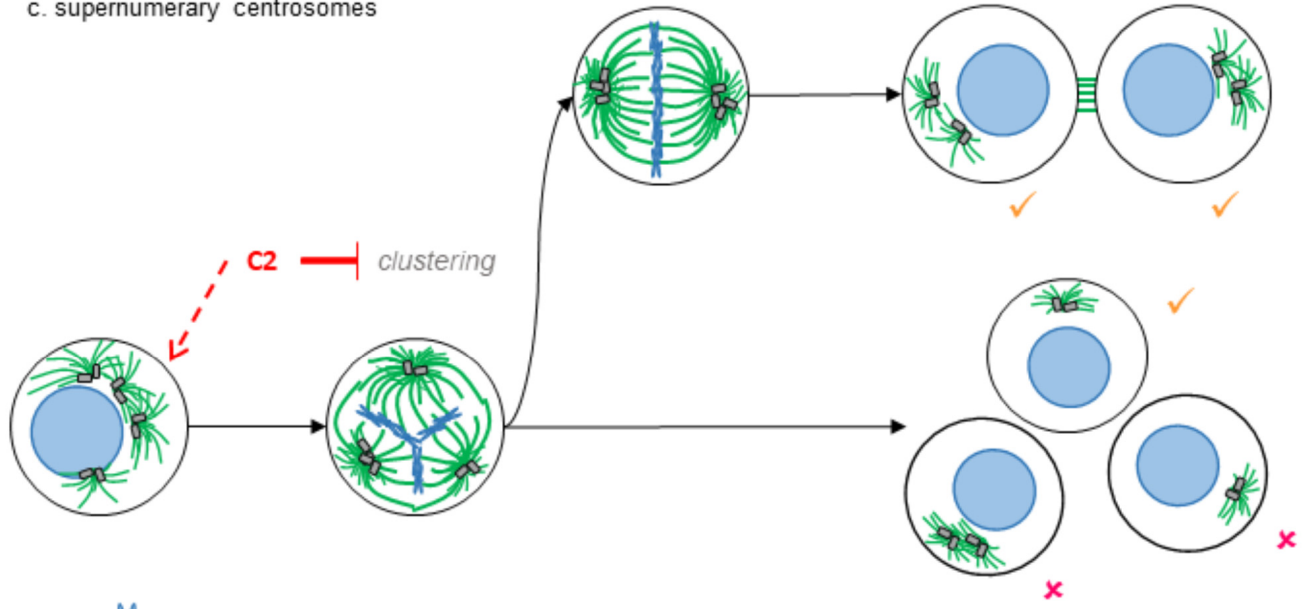

Centriole If Chromosome - Microtubule - Inhibition $-\rightarrow$ Induction

Figure 6. Model for the effects of the $\mathrm{C} 2$ combination on cell division. (a) Faithful chromosome segregation requires the formation of a bipolar spindle (green) that segregates the genetic material into two daughter cells (normal mitosis). Cancer cells are prone to form multipolar spindles due to fragmentation of the pericentriolar material or premature centriole splitting (b) or due to the presence of elevated centrosome numbers (c). Since multipolar spindles are often lethal, cancer cells rely on a spindle pole clustering mechanism to form pseudo-bipolar division for survival. Our results are consistent with the hypothesis that the $\mathbf{C} 2$ treatment prevents spindle pole clustering and potentially induces multipolar spindle formation, thus increasing the incidence of lethal multipolar cell divisions. 


\section{Discussion}

In this study, the phenotypically-driven TGMO-based screen was used to identify an optimal low-dose drug combination for ccRCC treatment with high efficacy and specificity towards a malignant clear cell-renal cell carcinoma 786-O cell line. This was achieved after the screening of only a small fraction of possible drug combinations in the entire search space of the drugs included in the screen. To increase specificity towards malignant cells, we introduced further constraints to our previously developed s-FSC method, which included (i) the parallel experimental testing in malignant and non-malignant cells to create opportunities for the therapeutic window (TW) and (ii) the inclusion of drug doses in the range of clinically attainable drug plasma concentrations, leading to the TGMO approach. Consequently, this resulted in the input drug dose of certain compounds (axitinib, VX-680, and sorafenib) that were even lower than the targeted $\mathrm{ED}_{20}$ [8]. Although counterintuitive to the optimization criteria, this reflects the heterogeneous treatment responses of different cell lines to clinically used drugs and the lack of efficacy of certain individual drug treatments at clinically relevant doses [31]. Modeling analysis of both efficacy (786-O cell viability inhibition) and the TW (difference between nonmalignant and malignant cells) led to the identification of a 786-O cell-specific and synergistic optimal drug combination (ODC) composed of CI-994, tubacin, erlotinib, and dasatinib (drug combination C2). C2 exhibited superior activity as compared to the current first-line standard ccRCC treatment (sunitinib).

When analyzing the regression models derived from the screen, the generated first-order terms clearly confirmed differences in mechanism and selectivity of the different drug classes included in the screen, i.e., HDACI and KI. While HDACI tended to have negative regression coefficients for efficacy in cancer and noncancerous cells, indicating a lack of selectivity towards malignant cells, KI generally gave positive regression coefficients in the TW indicating selective activity in cancer cells. Using the TW as an objective parameter for optimization, together with constraints on the drug doses limited by the maximally attainable clinical plasma drug concentrations, we identified a selective ODC with minimal activity in cross-validated nonmalignant cells.

The dose optimization step (Search 4) using both drug activity and the TW allowed for further increased efficacy and selectivity. These results support previous observations that in drug combinations, enhanced selectivity requires the use of multiple compounds (more than two) at optimized doses [32]. It has been proposed that the enhanced selectivity observed between synergizing drugs largely results from multitarget interactions, where one would expect synergies to be present in a relatively narrow spectrum of cellular phenotypes, increasing the chances of developing effective combinations without compromising the safety profile [2]. We proved that we can actively select a phenotype to be targeted, in our case the viability of cancer cells. Indeed, C2 optimized for cell viability inhibition did not necessarily present enhanced activity in the cell migration inhibition or endothelial network formation although it did show anti-angiogenic properties when tested in a developmental angiogenesis assay (i.e., the chicken embryo chorioallantoic membrane assay). Identified drug interactions included both antagonisms and synergies, guiding the selection of the most synergistic and potentiating drugs for the final optimized drug combination. Further refinement of the drug selection through Search 2 and Search 3 allowed for the confirmation of robust drug interactions, in particular the synergy between tubacin and erlotinib (Figure 1c-e). This is in line with the results of Liu et al. (2012) who demonstrated that HDAC6 treatment with trichostatin A or tubacin can effectively downregulate the activity of EGFR in the pdkd1-mutant epithelial cells, which in turn, results in the decreased phosphorylation of other targets downstream of EGFR, including ERK1/2 [33]. Furthermore, the VHL status of 786-O cells might also play a role in the synergy between erlotinib and tubacin in 786-O cells. The loss of VHL has been shown to extend the activation of EGFR and is associated with increased receptor half-life and retention in the endocytic pathway (the half-life time of EGFR is approx. $1.5 \mathrm{~h}$ in 786-O cells with VHL reconstitution and greater than $4 \mathrm{~h}$ in 786-O cells treated with TGF- $\alpha$ or EGF) [34], thus creating an important EGFR-dependent survival and growth advantage. These findings connect HDAC activity 
with EGFR inhibition and may partially explain the synergy between tubacin and erlotinib sensitizing the cells to apoptosis induction.

Acquired resistance to sunitinib is a common occurrence in the treatment of ccRCC [35,36]. It has recently been described that higher-order drug combinations may be needed to effectively target drug-resistant cells [37], which is further supported by the fact that the 786-O-specific C2 consists of four compounds. Importantly, C2 retained its anticancer efficacy in 786-OsunR cells, thus evidencing its potential to overcome cross-resistance mechanisms. This may suggest the need for higher-order drug combinations in targeting resistant cell types.

Based on our phenotypic analysis we postulate that the selective and synergistic anticancer efficacy of $\mathrm{C} 2$ in 786-O cells and MDA-MB-435 is based in part on its ability to favor persistent multipolar spindles. This is due to its ability to prevent spindle pole clustering, but the $\mathrm{C} 2$ treatment might also induce multipolar spindles on its own. Compounds that prevent pole clustering have emerged as a promising therapeutic agent, since they specifically target cancer cells that form multipolar spindles due to abnormal centrosome numbers, but do not affect mitotic progression in nonmalignant cells with normal centrosome numbers. Indeed, while normal cells as a rule have at mitotic onset 2 centrosomes with 2 centrioles, cancer cells frequently display abnormalities in centrosome organization, such as elevated centrosome numbers [26], which will impose transient multipolar spindles [38]. The minus-end directed kinesin HSET/KIF1C was the first promising target studied to develop new anticlustering drugs. As HSET depletion prevents clustering, it efficiently targets cancer cells with supernumerary centrosomes [39,40], yet it also induced multipolarity with acentrosomal poles [41]. So far, three HSET inhibitors have been reported: CW069, AZ82, and SR31527 [42-44], but their clinical efficacy has not yet been reported. We note that the phenotypes we observe with $\mathbf{C} 2$ treatment closely mirror the effects seen after HSET depletion. Indeed, C2 treatment prevents spindle pole clustering, and it might favor the formation of multipolar spindles, as at the first time point of our live cell imaging movies, we see more multipolar spindles. Alternatively, the lower percentage of initial multipolar spindles in DMSO-treated cells could be explained by rapid clustering within the first three minutes after NEBD.

We hypothesize that $\mathbf{C} 2$ targets cells prone to form multipolar spindles both in the context of abnormal centrosome/centriole numbers (MDA-MB-435) or in case of pericentriolar material fragmentation (786-O). This hypothesis is strengthened by our observation that $\mathbf{C} 2$ selectively targeted MDA-MB-435 cells, but not M14 cells, which generally have a normal set of centrosomes. Indeed, while elevated centrosome numbers are associated with mild chromosomal instability and cancer progression, persistent multipolar spindles with or without centrosomes are associated with massive chromosome segregation errors and cell death [45-49]. Moreover, we note that $\mathbf{C} 2$ treatment was also associated with micronuclei formation, which is prone to chromothripsis and loss of genetic information [50]. Nevertheless, we cannot exclude that apart from the ability to form multipolar spindles, other genetic differences between M14 and MDA-MB-435 cells play a role in determining the selective efficacy of the $\mathbf{C} 2$ treatment.

In particular, we note that in 786-O and 786-OsunR cells, C2 triggered cell death not only during mitosis, but also before cells could divide (Figures 3 and 4). This indicates that C2 also targets cancer cells via a mitosis-independent mechanism. Several declustering agents have been shown to target cells both in interphase and mitosis, yet their mechanism of action focuses on the disruption of the nuclear-centrosome-Golgi axis, which inhibits cell migration [51]. Since C2 did not affect cell migration, further investigation will be required to understand the $\mathbf{C} 2$ mechanism of action in interphase. We speculate that HDAC6 inhibition could play a critical role, as this enzyme is involved in ciliary disassembly [52], a mechanism causing cellular senescence when disrupted [53]. 


\section{Materials and Methods}

\subsection{Compounds}

CI-994, LBH-589, SAHA, axitinib, erlotinib HCl, BEZ-235, dasatinib, and sorafenib were purchased from LC Labs (Woburn, MA, USA), tubacin (SML0065) was purchased from Sigma-Aldrich (St. Louis, MO, USA) and VX-680 from Selleck Chemicals (Houston, TX, USA).

Compounds were dissolved in sterile DMSO (Sigma-Aldrich) at the following concentrations: CI-994 $(20 \mathrm{mg} / \mathrm{mL})$, LBH-589 $(5 \mathrm{mg} / \mathrm{mL})$, SAHA $(5 \mathrm{mg} / \mathrm{mL})$, tubacin $(5 \mathrm{mg} / \mathrm{mL})$, axitinib $(20 \mathrm{mg} / \mathrm{mL})$, erlotinib $\mathrm{HCl}(15 \mathrm{mg} / \mathrm{mL})$, BEZ-235 $(1 \mathrm{mg} / \mathrm{mL})$, dasatinib $(5 \mathrm{mg} / \mathrm{mL}), \mathrm{VX}-680(20 \mathrm{mg} / \mathrm{mL})$, and sorafenib $(40 \mathrm{mg} / \mathrm{mL})$. Aliquots were stored at $-80{ }^{\circ} \mathrm{C}$ and thawed prior to each experiment. A maximal concentration of $0.1 \%$ DMSO was allowed for any of the screened conditions and was used as a control (CTRL).

\subsection{Cells}

Human renal cell carcinoma cell line 786-O and human embryonic noncancerous HEK-293T cells were purchased from ATCC. Normal human dermal fibroblast adult (NHDF $\alpha$ ) cells were purchased from Lonza. The M14 and MDA435 cells were kindly gifted by Dr. M. Bettencourt-Dias. Immortalized ECRF24 endothelial cells macrovascular human endothelial cells immortalized (via immortalization procedures with amphotrophic replication-deficient retrovirus as described in [54]. Culture media consisted of RPMI for 786-O and HEK-293T cells, and DMEM for NHDF $\alpha$, M14 and MDA435 cells, supplemented with $10 \%$ of $5 \%$ fetal bovine serum and $1 \%$ penicillin/streptomycin (Life Technologies, Carlsbad, CA, USA). Cells have recently been tested for mycoplasma contamination and have been authenticated with PCR.

Human umbilical vein endothelial cells (HUVEC) were isolated from human umbilical cords and used for experiments only until passage 4. HUVEC cells were maintained in complete M199 medium (Gibco by ThermoFischer Scientific, Reinach, Switzerland) prepared freshly every week, supplemented with 10\% Fetal Bovine Serum (FBS), 1\% penicillin/streptomycin (ThermoFischer Scientific), $1 \%$ Endothelial Cell Growth supplement (ECGS; Millipore), $0.1 \mathrm{mg} / \mathrm{mL}$ heparin sodium salt (Sigma-Aldrich), $0.1 \mu \mathrm{M}$ Hydrocortisone (Sigma-Aldrich), and $10 \mu \mathrm{g} / \mathrm{mL}$ L-ascorbic acid (Stock $10 \mathrm{mg} / \mathrm{ml}$, dilute 1:1000, Sigma-Aldrich). HUVECs were grown in cell culture flasks coated with $0.2 \%$ gelatin (Sigma-Aldrich) and $1 \mathrm{mg} / \mathrm{mL}$ Collagen G (Biochrom AG, Berlin, Germany) in phosphate-buffered saline (PBS). Human vascular pericytes were purchased from ScienCell Research Laboratories and cultured according to the manufacturer's instructions. Pericytes were cultured in Pericyte Medium (ScienCell Research Laboratories, Carlsbad, CA, USA) supplemented with 2\% FBS, 1\% Pericyte Growth Supplement (ScienCell Research Laboratories), and 1\% penicillin/streptomycin solution (ScienCell Research Laboratories). The cells were grown in cell culture flasks coated with $15 \mu \mathrm{g} / \mathrm{mL}$ poly-L-lysine (Chemie Brunschwig AG, Basel, Switzerland) in $\mathrm{H}_{2} \mathrm{O}$.

786-OsunR cells were generated by chronically exposing ccRCC cell lines to sunitinib treatment $(1 \mu \mathrm{M})$. The response of cells to treatment was experimentally verified every two weeks. Cells were considered to show phenotypic resistance when the response of chronically exposure to sunitinib treatment was significantly reduced for at least one drug dose at or above the chronically administered dose.

\subsection{Cell Viability}

For cell viability experiments, cells were seeded in 96-well culture plates and $72 \mathrm{~h}$ treatments with $0.1 \%$ DMSO CTRL or drug (combinations) were initiated $24 \mathrm{~h}$ post-seeding. Seeding density was $2-10 \times 10^{3}$ cells/well, depending on the cell line's growth characteristics such that the control cells approach confluence at the time of the assay readout. Subsequently, cell viability was assessed using the CellTiter-Glo ${ }^{\circledR}$ luminescence assay (Promega, Madison, WI, USA) and cell viability was reported as the luminescence signal in the treated wells normalized to the signal in the DMSO treated 
control wells [55]. Dose-response curves and $\mathrm{IC}_{50}$ values were determined in Graphpad Prism ${ }^{\circledR}$ using a four-parameter nonlinear fit of the log-transformed dose data of each compound.

\subsection{Data Modeling and Analysis for Combinatorial Drug Screening}

The TGMO platform was applied as previously described for s-FSC [8]. Briefly, drug combinations were selected based on a design of experiment (DoE) using the orthogonal array composite design (OACD) [12]. The activity of each drug combination was experimentally defined in vitro cell metabolic activity assays using therapeutic window and including maximal tolerated dose of each drug in human (Supplementary Figure S2a) and results were modeled in Matlab ${ }^{\circledR}$ using second-order step-wise linear regression models. The accuracy and reliability of regression models was confirmed based on analysis including the elimination of outlier data points (based on measurements of Cook's distance), the evaluation of the coefficient of multiple determination $\left(R^{2}\right)$ and the root mean square error (RMSE) and the correlations between fitted and observed data points (Supplementary Figure S2b), as well as through the visualization of residual plots, Cook's distance plot, normal Q-Q plot and residual histogram [12] (Supplementary Figure S2c). Finally, an ANOVA lack of fit test was performed to confirm the selection of an appropriate model structure. Higher-order terms, such as three-drug interactions, are generally considered to make a negligible contribution to overall activity and are therefore not included in the analysis [56]. Regression models were used to eliminate compounds from the search, leading the subsequent search round, composed of the experimental exploration of a smaller subset of drug combinations defined by an OACD design and analysis based on regression modeling. Thus, the TGMO platform was applied in 3 sequential search rounds. The dose-optimization search (Search 4) was achieved with the final selection of 4 compounds performed in an expanded dose range.

\subsection{Three-Dimensional Cell Cultures}

The efficacy of $\mathbf{C 2}$ and corresponding monotherapies was evaluated in homotypic 786-O, seeded 3000 cells/well, and heterotypic three-dimensional (3D) models. The latter model consisted of 3000 786-O cells cocultured in a 1:1 ratio with 3000 NHDF $\alpha$ cells, and 600 freshly isolated HUVEC cells (corresponding to $10 \%$ ). The cell mixture was supplemented with $2.5 \%$ matrigel (Corning matrigel, \#354230) and $50 \mu \mathrm{L} /$ well was seeded in round bottom 96-well plates with a cell-repellent surface (Cellstar ${ }^{\circledR}$ 650970) by using an electronic multidispensing pipette at low speed. The plate was centrifuged at $400 \times \mathrm{g}$ at $4{ }^{\circ} \mathrm{C}$. The whole procedure was performed on ice and materials were precooled to avoid polymerization of matrigel. The cocultured spheroids were incubated with drugs for $72 \mathrm{~h}$. Cell viability was measured with the CellTiter-Glo ${ }^{\circledR}$ 3D Cell Viability Assay (Promega, \#G9681). Luminescent signals were measured with a luminescent plate reader (Biotek Cytation 3 with corresponding software at the standard settings).

\subsection{Two-Dimensional Cell Migration Assay}

The 2-dimensional cell migration assay was performed in an endothelial cell scratch assay [57]. 786-O cells were seeded at a density of 3-3.5 $\times 10^{4}$ cells/well) cells in a 96-well cell culture plate and grown overnight. A scratch was made using a sterile scratch tool (Peira Scientific Instruments, Beerse, Belgium) and the treatment was administered immediately after. The scratch wounds were imaged using a Leica DMI3000 microscope (Leica, Rijswijk, The Netherlands) at $\times 5$ magnification using Universal Grab 6.3 software (DCILabs, Keerbergen, Belgium). Imaging was performed at $\mathrm{T}=0 \mathrm{~h}$ and several time points after scratching until $\mathrm{T}=7 \mathrm{~h}$. The size of the scratch was automatically quantified and analyzed using Scratch Assay 6.2 (DCILabs) software by calculating the absolute wound closure (initial minus final scratch surface) [58].

\subsection{Immunofluorescence}

Cells were seeded on glass in a 6-well plate with a density of 100,000 cells/well for 786-O, 786-OsunR and MDA435 150,000 cells/well for M14 cells the day prior to the experiment. To investigate 
the cause of multipolarity, cells were treated for $12 \mathrm{H}$ with DMSO $0.1 \%$ or $\mathbf{C} 2$, then with $1 \mu \mathrm{g} / \mathrm{ml}$ nocodazole (Sigma, Buchs, Switzerland) for $30 \mathrm{~min}$. Normal medium was then added to the cells for $15 \mathrm{~min}$ before fixation. Cells were fixed with methanol at $-20{ }^{\circ} \mathrm{C}$ for $6 \mathrm{~min}$, rinsed with PBS, blocked with 3\% BSA RT, then stained for $1 \mathrm{~h}$ RT. The following primary antibodies were used: human anti $\alpha$-tubulin (1:50, this study), rabbit serum anti- $\gamma$ tubulin (1:2000, this study), and mouse anti-centrin (1:2000, Merck Millipore, clone 20H5, Buchs, Switzerland). The secondary antibodies (Invitrogen, Zug, Switzerland) used were Alexa Fluor 488 anti-mouse (A21202), Alexa Fluor 594 anti-rabbit (A11012), Alexa Fluor 568 anti-rabbit (A11036), and Alexa Fluor 647 anti-human (A21445), diluted 1:400 and incubated for $30 \mathrm{~min}$ at RT on cells. Coverslips were mounted with VECTASHIELD with DAPI (Vector Laboratories, Burlingame, CA, USA). Z-stack images were taken using an Olympus DeltaVision wide-field microscope (GE Healthcare, Chicago, IL, USA) with $60 \times 1.4$ NA oil objective and recorded with a Coolsnap HQ2 CCD camera (Roper Scientific, Martinsried, Germany) and the Softworx software (GE Healthcare) with $0.2 \mu \mathrm{m}$ spacing between stacks.

\subsection{Mitosis Live Cell Imaging}

To visually monitor the effect of the drug combination $\mathrm{C} 2$ on 786-O cells, live-cell imaging was performed for $24 \mathrm{~h}$ at $37^{\circ} \mathrm{C}$ on the Ti widefield microscope (Nikon, Egg, Switzerland) equipped with an environmental chamber using either a $60 \times 1.3 \mathrm{NA}$ or a $40 \times 1.3$ oil objective and a CoolSNAP HQ camera (Roper Scientific, Tucson, AZ, USA). The sampling rate was 3 min with recording at each time point of 9 Z-stacks separated by $2 \mu \mathrm{m}$ and 20 points were taken per condition. Two hours before the start of the movie, SiR-Tubulin was added on the cells $(50 \mathrm{nM}$, SpiroChrome AG, Stein am Rhein, Switzerland) to stain microtubules, with Valspodar $(4 \mu \mathrm{M})$ to keep the dye inside the cells. Time-lapse movies were analyzed using NIS Elements AR Software and cropped into figures using ImageJ FIJI software and Adobe Illustrator CC.

\subsection{Statistical Analysis}

If not indicated differently, the data is presented as the mean of multiple independent experiments and error bars represent the standard error of the mean, unless otherwise specified. Significance was determined using the one-way or two-way ANOVA test with a post hoc multiple comparison test or a Student's T-test (Graphpad Prism ${ }^{\circledR}$ ). ${ }^{*} p<0.05$ and ${ }^{* *} p<0.01$ were considered statistically significant and are indicated versus the control or single-drug treatment groups, as specified in figure legends.

\section{Conclusions}

In summary, we discovered the low-dose synergistic drug combination $\mathbf{C} 2$ that has the ability to inhibit multipolar spindle pole clustering in renal cell carcinoma 786-O and melanoma cells with high centrosome amplification. C2 appears to selectively target a survival mechanism employed by cancer cells, promoting multipolar division and generation of nonproliferative daughter cells with compromised viability while showing negligible activity in nonmalignant cell types. Understanding the full mechanism of action of $\mathbf{C} 2$ and testing its activity in various cancer types will allow analyzing whether the presence of multipolar spindles is a sole biomarker for $\mathbf{C} 2$ treatment.

Supplementary Materials: The following are available online at http://www.mdpi.com/2072-6694/11/10/1612/s1. Figure S1: 786-O and HEK-293T dose-response curves for all drugs included in the TGMO-based screen. Figure S2: Overview of optimization procedure and results of first search round in TGMO. Figure S3: Results of drug combination optimization. Figure S4: Efficacy of non-optimized drug combinations screened in Search 1 and composition of corresponding drug combinations. Figure S5: Anti-angiogenic activity of the optimized drug combinations. Figure S6: 786-O cell cycle and cell morphology are influenced by C2 treatment. Figure S7: Efficacy of optimized drug combinations on 786-O 2D migration. Figure S8: C2 does not affect HEK-293T cells. Figure S9: Sunitinib resistance induced in 786-O cells.

Author Contributions: Conceptualization, P.N.-S., A.W.; Methodology, P.N.-S., A.W., M.L.R.-B., P.M., M.Z., M.R., D.H., M.M.-L., K.F., B.W.-H.; Software, A.W., M.L.R.-B., M.Z., M.R., G.M.R.; Validation, A.W., M.Z., M.L.R.-B., M.R., K.F., D.H., G.M.R., B.W.-H.; Formal Analysis, A.W., M.L.R.-B., M.Z., M.R., K.F., D.H., G.M.R., M.M.-L., 
B.W.-H., P.M., P.N.-S.; Investigation, A.W., M.Z., M.L.R.-B., M.R., K.F., D.H., G.M.R., M.M.-L., B.W.-H., P.M., P.N.-S.; Resources, P.N.-S.; Data Curation, A.W., M.Z., M.L.R.-B., M.R., K.F., D.H., B.W.-H., P.N.-S.; Writing-A.W., M.L.R.-B., P.N.-S.; Writing-Review \& Editing, A.W., M.Z., M.L.R.-B., M.R., K.F., D.H., G.M.R., M.M.L., B.W.-H., P.M., P.N.-S.; Visualization, A.W., M.L.R.-B., M.Z., M.R.; Supervision, P.N.-S. and P.M.; Project Administration, P.N.-S.; Funding Acquisition, P.N.-S.

Funding: This work was funded by European Research Council (680209 to PNS) and Novartis Foundation of Medical-Biological Research (17A003 to PNS).

Acknowledgments: The authors acknowledge Claudia Simões-Pires for her expert opinion and Tse Wong and Emilie Besseling for expert technical support.

Conflicts of Interest: The authors are the inventors on pending (PNS, PM, AW, MLRB, EP19199136) and issued (PNS, AW, WO2015136061A3) patents on methods of drug combination therapy.

\section{References}

1. Bozic, I.; Reiter, J.G.; Allen, B.; Antal, T.; Chatterjee, K.; Shah, P.; Moon, Y.S.; Yaqubie, A.; Kelly, N.; Le, D.T.; et al. Evolutionary Dynamics of Cancer in Response to Targeted Combination Therapy. Elife 2013, 2, 747. [CrossRef]

2. Lehar, J.; Krueger, A.S.; Avery, W.; Heilbut, A.M.; Johansen, L.M.; Price, E.R.; Rickles, R.J.; Short, G.F., 3rd; Staunton, J.E.; Jin, X.; et al. Synergistic Drug Combinations Tend to Improve Therapeutically Relevant Selectivity. Nat. Biotechnol. 2009, 27, 659-666. [CrossRef] [PubMed]

3. Keith, C.T.; Borisy, A.A.; Stockwell, B.R. Multicomponent Therapeutics for Networked Systems. Nat. Rev. Drug Discov. 2005, 4, 71-78. [CrossRef] [PubMed]

4. Kitano, H. A Robustness-Based Approach to Systems-Oriented Drug Design. Nat. Rev. Drug Discov. 2007, 6, 202-210. [CrossRef] [PubMed]

5. Lehár, J.; Krueger, A.; Zimmermann, G.; Borisy, A. High-Order Combination Effects and Biological Robustness. Mol. Syst. Biol. 2008, 4, 215. [CrossRef] [PubMed]

6. Delaney, J.R.; Patel, C.; McCabe, K.E.; Lu, D.; Davis, M.A.; Tancioni, I.; von Schalscha, T.; Bartakova, A.; Haft, C.; Schlaepfer, D.D.; et al. A Strategy to Combine Pathway-Targeted Low Toxicity Drugs in Ovarian Cancer. Oncotarget 2015, 6, 31104-31118. [CrossRef]

7. Liu, S.; Nikanjam, M.; Kurzrock, R. Dosing de Novo Combinations of Two Targeted Drugs: Towards A Customized Precision Medicine Approach to Advanced Cancers. Oncotarget 2016, 7, 11310-11320. [CrossRef]

8. Weiss, A.; Berndsen, R.H.; Ding, X.; Ho, C.M.; Dyson, P.J.; van den Bergh, H.; Griffioen, A.W.; Nowak-Sliwinska, P. A Streamlined Search Technology for Identification of Synergistic Drug Combinations. Sci. Rep. 2015, 5, 14508. [CrossRef]

9. Weiss, A.; Nowak-Sliwinska, P. Current Trends in Multidrug Optimization: An Alley of Future Successful Treatment of Complex Disorders. SLAS Technol. 2017, 22, 254-275. [CrossRef]

10. Nowak-Sliwinska, P.; Weiss, A.; Ding, X.; Dyson, P.J.; van den Bergh, H.; Griffioen, A.W.; Ho, C.M. Optimization of Drug Combinations Using Feedback System Control. Nat. Protoc. 2016, 11, 302-315. [CrossRef]

11. Ding, X.; Liu, W.; Weiss, A.; Li, Y.; Wong, I.; Griffioen, A.W.; van den Bergh, H.; Xu, H.; Nowak-Sliwinska, P.; Ho, C.M.; et al. Discovery of A Low Order Drug-Cell Response Surface for Applications in Personalized Medicine. Phys. Biol. 2014, 11, 065003. [CrossRef] [PubMed]

12. Xu, H.; Jaynes, J.; Ding, X. Combinig Two-Level and Three-Level Orthogonal Arrays for Factor Screening and Response Surface Exploration. Stat. Sin. 2014, 24, 269-289.

13. Baldewijns, M.M.; van Vlodrop, I.J.; Schouten, L.J.; Soetekouw, P.M.; de Bruïne, A.P.; van Engeland, M. Genetics and Epigenetics of Renal Cell Cancer. Biochim. Biophys. Acta 2008, 1785, 133-155. [CrossRef] [PubMed]

14. Marcus, A.I.; Zhou, J.; O’Brate, A.; Hamel, E.; Wong, J.; Nivens, M.; El-Naggar, A.; Yao, T.P.; Khuri, F.R.; Giannakakou, P. The Synergistic Combination of the Farnesyl Transferase Inhibitor Lonafarnib and Paclitaxel Enhances Tubulin Acetylation and Requires a Functional Tubulin Deacetylase. Cancer Res. 2005, 65, 3883-3893. [CrossRef] [PubMed]

15. LaBonte, M.J.; Wilson, P.M.; Fazzone, W.; Russell, J.; Louie, S.G.; El-Khoueiry, A.; Lenz, H.J.; Ladner, R.D. The Dual EGFR/HER2 Inhibitor Lapatinib Synergistically Enhances the Antitumor Activity of the Histone Deacetylase Inhibitor Panobinostat in Colorectal Cancer Models. Cancer Res. 2011, 71, 3635-3648. [CrossRef] 
16. Chen, M.C.; Chen, C.H.; Wang, J.C.; Tsai, A.C.; Liou, J.P.; Pan, S.L.; Teng, C.M. The HDAC Inhibitor, MPT0E028, Enhances Erlotinib-Induced Cell Death in EGFR-TKI-Resistant NSCLC Cells. Cell Death Dis. 2013, 4, 810. [CrossRef]

17. Thurn, K.T.; Thomas, S.; Moore, A.; Munster, P.N. Rational Therapeutic Combinations with Histone Deacetylase Inhibitors for the Treatment of Cancer. Future Oncol. 2011, 7, 263-283. [CrossRef]

18. Berndsen, R.H.; Abdul, U.K.; Weiss, A.; Zoetemelk, M.; Te Winkel, M.T.; Dyson, P.J.; Griffioen, A.W.; Nowak-Sliwinska, P. Epigenetic Approach for Angiostatic Therapy: Promising Combinations for Cancer Treatment. Angiogenesis 2017, 20, 245-267. [CrossRef]

19. Chou, T.C. Drug combination studies and their synergy quantification using the chou-talalay method. Cancer Res 2010, 70, 440-446. [CrossRef]

20. Nowak-Sliwinska, P.; Alitalo, K.; Allen, E.; Anisimov, A.; Aplin, A.C.; Auerbach, R.; Augustin, H.G.; Bates, D.O.; van Beijnum, J.R.; Bender, R.H.F.; et al. Consensus Guidelines for the Use and Interpretation of Angiogenesis Assays. Angiogenesis 2018, 21, 425-532. [CrossRef]

21. Nowak-Sliwinska, P.; Segura, T.; Iruela-Arispe, M.L. The Chicken Chorioallantoic Membrane Model in Biology, Medicine and Bioengineering. Angiogenesis 2014, 17, 779-804. [CrossRef] [PubMed]

22. Leibowitz, M.L.; Zhang, C.Z.; Pellman, D. Chromothripsis: A New Mechanism for Rapid Karyotype Evolution. Annu. Rev. Genet. 2015, 49, 183-211. [CrossRef] [PubMed]

23. Normand, G.; King, R.W. Understanding Cytokinesis Failure. Adv. Exp. Med. Biol. 2010, 676, $27-55$. [PubMed]

24. Fu, J.; Hagan, I.M.; Glover, D.M. The Centrosome and its Duplication Cycle. Cold Spring Harb. Perspect. Biol. 2015, 7, 15800. [CrossRef]

25. Nigg, E.A. Centrosome Aberrations: Cause or Consequence of Cancer Progression? Nat. Rev. Cancer 2002, 2, 815-825. [CrossRef]

26. Godinho, S.A.; Pellman, D. Causes and Consequences of Centrosome Abnormalities in Cancer. Philos. Trans. R. Soc. Lond. B Biol. Sci. 2014, 369. [CrossRef]

27. Adar, Y.; Stark, M.; Bram, E.E.; Nowak-Sliwinska, P.; van den Bergh, H.; Szewczyk, G.; Sarna, T.; Skladanowski, A.; Griffioen, A.W.; Assaraf, Y.G. Imidazoacridinone-Dependent Lysosomal Photodestruction: A Pharmacological Trojan Horse Approach to Eradicate Multidrug-Resistant Cancers. Cell Death Dis. 2012, 3 , 1-10. [CrossRef]

28. Nigg, E.A.; Stearns, T. The Centrosome Cycle: Centriole Biogenesis, Duplication and Inherent Asymmetries. Nat. Cell Biol. 2011, 13, 1154-1160. [CrossRef]

29. Zyss, D.; Gergely, F. Centrosome Function in Cancer: Guilty or Innocent? Trends Cell Biol. 2009, 19, 334-346. [CrossRef]

30. Marteil, G.; Guerrero, A.; Vieira, A.F.; de Almeida, B.P.; Machado, P.; Mendonça, S.; Mesquita, M.; Villarreal, B.; Fonseca, I.; Francia, M.E.; et al. Over-Elongation of Centrioles in Cancer Promotes Centriole Amplification and Chromosome Missegregation. Nat. Commun. 2018, 9, 1258. [CrossRef]

31. Crusz, S.M.; Tang, Y.Z.; Sarker, S.J.; Prevoo, W.; Kiyani, I.; Beltran, L.; Peters, J.; Sahdev, A.; Bex, A.; Powles, T.; et al. Heterogeneous Response and Progression Patterns Reveal Phenotypic Heterogeneity of Tyrosine Kinase Inhibitor Response in Metastatic Renal Cell Carcinoma. BMC Med. 2016, 14, 185. [CrossRef] [PubMed]

32. Kashif, M.; Andersson, C.; Hassan, S.; Karlsson, H.; Senkowski, W.; Fryknäs, M.; Nygren, P.; Larsson, R.; Gustafsson, M.G. In Vitro Discovery of Promising Anti-Cancer Drug Combinations Using Iterative Maximisation of a Therapeutic Index. Sci. Rep. 2015, 5, 14118. [CrossRef] [PubMed]

33. Liu, W.; Fan, L.X.; Zhou, X.; Sweeney, W.E., Jr.; Avner, E.D.; Li, X. HDAC6 Regulates Epidermal Growth Factor Receptor (EGFR) Endocytic Trafficking and Degradation in Renal Epithelial Cells. PLoS ONE 2012, 7, 49418. [CrossRef] [PubMed]

34. Wang, Y.; Roche, O.; Yan, M.S.; Finak, G.; Evans, A.J.; Metcalf, J.L.; Hast, B.E.; Hanna, S.C.; Wondergem, B.; Furge, K.A.; et al. Regulation of Endocytosis via the Oxygen-Sensing Pathway. Nat. Med. 2009, 15, 319-324. [CrossRef] [PubMed]

35. Molina, A.M.; Lin, X.; Korytowsky, B.; Matczak, E.; Lechuga, M.J.; Wiltshire, R.; Motzer, R.J. Sunitinib Objective Response in Metastatic Renal Cell Carcinoma: Analysis of 1059 Patients Treated on Clinical Trials. Eur. J. Cancer 2014, 50, 351-358. [CrossRef] 
36. Joosten, S.C.; Hamming, L.; Soetekouw, P.M.; Aarts, M.J.; Veeck, J.; van Engeland, M.; Tjan-Heijnen, V.C. Resistance to Sunitinib in Renal Cell Carcinoma: From Molecular Mechanisms to Predictive Markers and Future Perspectives. Biochim. Biophys. Acta 2015, 1855, 1-16. [CrossRef]

37. Horn, T.; Ferretti, S.; Ebel, N.; Tam, A.; Ho, S.; Harbinski, F.; Farsidjani, A.; Zubrowski, M.; Sellers, W.R.; Schlegel, R.; et al. High Order Drug Combinations are Required to Effectively Kill Colorectal Cancer Cells. Cancer Res. 2016, 76, 6950-6963. [CrossRef]

38. Meraldi, P. Centrosomes in Spindle Organization and Chromosome Segregation: A Mechanistic View. Chromosome Res. 2016, 24, 19-34. [CrossRef]

39. Kwon, M.; Godinho, S.A.; Chandhok, N.S.; Ganem, N.J.; Azioune, A.; Thery, M.; Pellman, D. Mechanisms to Suppress Multipolar Divisions in Cancer Cells with Extra Centrosomes. Genes Dev. 2008, 22, 2189-2203. [CrossRef]

40. Chavali, P.L.; Chandrasekaran, G.; Barr, A.R.; Tátrai, P.; Taylor, C.; Papachristou, E.K.; Woods, C.G.; Chavali, S.; Gergely, F. A CEP215-HSET Complex Links Centrosomes with Spindle Poles and Drives Centrosome Clustering in Cancer. Nat. Commun. 2016, 7, 11005. [CrossRef]

41. Kleylein-Sohn, J.; Pöllinger, B.; Ohmer, M.; Hofmann, F.; Nigg, E.A.; Hemmings, B.A.; Wartmann, M. Acentrosomal Spindle Organization Renders Cancer Cells Dependent on the Kinesin HSET. J. Cell Sci. 2012, 125, 5391-5402. [CrossRef] [PubMed]

42. Wu, J.; Mikule, K.; Wang, W.; Su, N.; Petteruti, P.; Gharahdaghi, F.; Code, E.; Zhu, X.; Jacques, K.; Lai, Z.; et al. Discovery and Mechanistic Study of A Small Molecule Inhibitor for Motor Protein KIFC1. ACS Chem. Biol. 2013, 8, 2201-2208. [CrossRef] [PubMed]

43. Zhang, W.; Zhai, L.; Wang, Y.; Boohaker, R.J.; Lu, W.; Gupta, V.V.; Padmalayam, I.; Bostwick, R.J.; White, E.L.; Ross, L.J.; et al. Discovery of A Novel Inhibitor of Kinesin-Like Protein KIFC1. Biochem. J. 2016, 473, 1027-1035. [CrossRef] [PubMed]

44. Watts, C.A.; Richards, F.M.; Bender, A.; Bond, P.J.; Korb, O.; Kern, O.; Riddick, M.; Owen, P.; Myers, R.M.; Raff, J.; et al. Design, Synthesis, and Biological Evaluation of An Allosteric Inhibitor of HSET that Targets Cancer Cells with Supernumerary Centrosomes. Chem. Biol. 2013, 20, 1399-1410. [CrossRef] [PubMed]

45. Ganem, N.J.; Godinho, S.A.; Pellman, D. A Mechanism Linking Extra Centrosomes to Chromosomal Instability. Nature 2009, 460, 278-282. [CrossRef] [PubMed]

46. Silkworth, W.T.; Nardi, I.K.; Scholl, L.M.; Cimini, D. Multipolar Spindle Pole Coalescence is A Major Source of Kinetochore Mis-Attachment and Chromosome Mis-Segregation in Cancer Cells. PLoS ONE 2009, 4, 6564. [CrossRef] [PubMed]

47. Sercin, O.; Larsimont, J.C.; Karambelas, A.E.; Marthiens, V.; Moers, V.; Boeckx, B.; Le Mercier, M.; Lambrechts, D.; Basto, R.; Blanpain, C. Transient PLK4 Overexpression Accelerates Tumorigenesis in p53-Deficient Epidermis. Nat. Cell Biol. 2016, 18, 100-110. [CrossRef]

48. Levine, M.S.; Bakker, B.; Boeckx, B.; Moyett, J.; Lu, J.; Vitre, B.; Spierings, D.C.; Lansdorp, P.M.; Cleveland, D.W.; Lambrechts, D.; et al. Centrosome Amplification Is Sufficient to Promote Spontaneous Tumorigenesis in Mammals. Dev. cell 2017, 40, 313-322. [CrossRef]

49. Zasadil, L.M.; Andersen, K.A.; Yeum, D.; Rocque, G.B.; Wilke, L.G.; Tevaarwerk, A.J.; Raines, R.T.; Burkard, M.E.; Weaver, B.A. Cytotoxicity of Paclitaxel in Breast Cancer is Due to Chromosome Missegregation on Multipolar Spindles. Sci. Transl. Med. 2014, 6, 229ra43. [CrossRef]

50. Crasta, K.; Ganem, N.J.; Dagher, R.; Lantermann, A.B.; Ivanova, E.V.; Pan, Y.; Nezi, L.; Protopopov, A.; Chowdhury, D.; Pellman, D. DNA Breaks and Chromosome Pulverization from Errors in Mitosis. Nature 2012, 482, 53-58. [CrossRef]

51. Pannu, V.; Rida, P.C.; Celik, B.; Turaga, R.C.; Ogden, A.; Cantuaria, G.; Gopalakrishnan, J.; Aneja, R. Centrosome-Declustering Drugs Mediate A Two-Pronged Attack on Interphase and Mitosis in Supercentrosomal Cancer Cells. Cell Death Dis. 2014, 5, 1538. [CrossRef] [PubMed]

52. Ran, J.; Yang, Y.; Li, D.; Liu, M.; Zhou, J. Deacetylation of Alpha-Tubulin and Cortactin is Required for HDAC6 to Trigger Ciliary Disassembly. Sci. Rep. 2015, 5, 12917. [CrossRef] [PubMed]

53. Jeffries, E.P.; Di Filippo, M.; Galbiati, F. Failure to Reabsorb the Primary Cilium Induces Cellular Senescence. FASEB J. 2018, 33, 4866-4882. [CrossRef] [PubMed] 
54. Fontijn, R.; Hop, C.; Brinkman, H.J.; Slater, R.; Westerveld, A.; van Mourik, J.A.; Pannekoek, H. Maintenance of Vascular Endothelial Cell-Specific Properties after Immortalization with An Amphotrophic Replication-Deficient Retrovirus Containing Human Papilloma Virus 16 E6/E7 DNA. Exp. Cell Res. 1995, 216, 199-207. [CrossRef] [PubMed]

55. Berndsen, R.H.; Swier, N.; van Beijnum, J.R.; Nowak-Sliwinska, P. Colorectal Carcinoma Growth Retardation through Induction of Apoptosis, Using An Optimized Synergistic Cocktail of Axitinib, Erlotinib and Dasatinib. Cancers 2019. under revisions.

56. Wood, K.; Nishida, S.; Sontag, E.D.; Cluzel, P. Mechanism-Independent Method for Predicting Response to Multidrug Combinations in Bacteria. Proc. Natl. Acad. Sci. USA 2012, 109, 12254-12259. [CrossRef]

57. Van Beijnum, J.R.; Nowak-Sliwinska, P.; van Berkel, M.; Wong, T.J.; Griffioen, A.W. A Genomic Screen for Angiosuppressor Genes in the Tumor Endothelium Identifies A Multifaceted Angiostatic Role for Bromodomain Containing 7 (BRD7). Angiogenesis 2017, 20, 641-654. [CrossRef]

58. Van Beijnum, J.R.; Nowak-Sliwinska, P.; van den Boezem, E.; Hautvast, P.; Buurman, W.A.; Griffioen, A.W. Tumor Angiogenesis is Enforced by Autocrine Regulation of High-Mobility Group Box 1. Oncogene 2013, 17, 363-374. [CrossRef]

(C) 2019 by the authors. Licensee MDPI, Basel, Switzerland. This article is an open access article distributed under the terms and conditions of the Creative Commons Attribution (CC BY) license (http://creativecommons.org/licenses/by/4.0/). 